\title{
In the Era of Therapeutic Hypothermia, How Well Do Studies of Perinatal Neuroprotection Control Temperature?
}

\author{
Robert Galinsky a, b Justin M. Dean ${ }^{a} \quad$ Christopher A. Lear ${ }^{a} \quad$ Joanne O. Davidson ${ }^{a}$ \\ Simerdeep Dhillon ${ }^{a}$ Guido Wassink $^{\mathrm{a}}$ Laura Bennet $^{\mathrm{a}}$ Alistair J. Gunn ${ }^{\mathrm{a}}$ \\ a Department of Physiology, Faculty of Medical and Health Sciences, University of Auckland, Auckland, \\ New Zealand; ${ }^{b}$ The Ritchie Centre, Hudson Institute of Medical Research, Clayton, VIC, Australia
}

\section{Keywords}

Perinatal brain injury · Cerebral palsy · Hypoxic-ischemic encephalopathy · Asphyxia · Neuroprotection

\begin{abstract}
In the era of therapeutic hypothermia, reliable preclinical studies are integral to successfully identify neuroprotective strategies to further improve outcomes of encephalopathy at term. We reviewed preclinical neuroprotection studies reported between January 2014 and June 2016 to assess the use of effective temperature monitoring and control. As a secondary measure, we examined whether studies addressed other methodological issues such as stage of brain development, sex differences, the timing of the treatment relative to the insult, and the histological and functional endpoints used after hypoxia-ischemia. The extent and duration of temperature monitoring was highly inconsistent. Only a minority of papers monitored core $(19 / 61 ; 31 \%)$ or brain temperature $(3 / 61 ; 5 \%)$. Most $(40 / 45)$ of the neuroprotectants either were likely to affect thermoregulation or their impact is unknown. In $85 \%$ of papers neonatal rodents were used ( $67 \%$ at $P 7) ; 51 \%$ of papers did not report the sex of the animals or tested the effect of potential neuroprotectants on just one sex. In $76 \%$ of studies, treatment was before or im-
\end{abstract}

\section{KARGER}

() 2016 S. Karger AG, Basel

E-Mail karger@karger.com

www.karger.com/dne mediately after the insult (within the first $2 \mathrm{~h}$ ), and few studies assessed long-term histological and behavioral outcomes. In conclusion, many recent preclinical neonatal studies cannot exclude the possibility that apparent neuroprotection might be related to drug-induced hypothermia or to other methodological choices. Close monitoring and control of brain temperature during, as well as for many days after, experimental hypoxia-ischemia are now critical to reliably develop new ways to improve neurodevelopmental outcomes after perinatal hypoxic-ischemic encephalopathy.

(c) 2016 S. Karger AG, Basel

\section{Introduction}

Mild "therapeutic" hypothermia for hypoxic-ischemic encephalopathy (HIE) in near-term and term infants is now well established to improve survival without disability [1]. Current hypothermia protocols are only partially protective, such that nearly half of infants still die or survive with disability despite cooling [2], and so it is vital to identify adjunct therapies to further improve outcomes from HIE [3]. The corollary of the successful clinical translation of therapeutic hypothermia is that we can now be certain that even small changes in brain and/or body

Prof. Alistair J. Gunn, MBChB, $\mathrm{PhD}$

Department of Physiology, Faculty of Medical and Health Sciences University of Auckland

Private Bag 92019, Auckland 1023 (New Zealand)

E-Mail aj.gunn@auckland.ac.nz 
temperature critically affect outcome in both animals [47] and humans [8-10]. Indeed, many apparent neuroprotective effects of various pharmacological agents can be mediated by drug-induced hypothermia, as previously reviewed [11]. Conversely, neuroprotection can be masked by delayed hyperthermia [6].

Neonatal rodents are widely used to model HIE and assess neuroprotective strategies. However, due to their small body mass relative to surface area, lack of subcutaneous fat, naked skin, poor control of peripheral vasculature, and absent shivering [12], the infant rat produces less heat and loses more body heat than adults [13]. These factors make them functionally poikilothermic and particularly susceptible to rapid changes in body and brain temperature during changes in environmental temperature [14]. Further, their brains are relatively small and flat, which can allow selective brain cooling, particularly if blood flow is reduced after HI [11]. Behavioral thermoregulation in neonatal rodents begins to emerge during the 1st week of life, primarily through metabolism of brown adipose tissue and group huddling [15]. However, their thermoregulatory ability is not fully developed until the 3 rd week of life [16].

Thus, in this study, we reviewed studies reported between January 2014 and June 2016 to assess the use of effective temperature monitoring and control in preclinical neuroprotection studies in the era of therapeutic hypothermia. As a secondary measure, we examined whether these studies addressed other important methodological issues [17], such as the stage of brain development, sex differences, the timing of the treatment relative to the insult, and the duration of follow-up for histological and functional endpoints after $\mathrm{HI}$.

\section{Analysis Strategy}

In this review, a group of researchers focusing on perinatal neuroprotection have examined preclinical studies of perinatal neuroprotection reported from January 1, 2014 , to June 30,2016 , for how well these studies control for temperature in near-term or term-equivalent brain injury. The studies were grouped into those that demonstrated improved or unaffected neuropathological or neurodevelopmental outcomes. Studies were further subdivided by species, type of insult used to induce HIE, the treatment used, timing of treatment relative to the insult, extent of temperature monitoring, sex of the subjects, main study outcomes (histological and/or functional), and survival time after the insult based on histological and/or functional assessments. Studies were searched for on PubMed, MEDLINE (OvidSP), and Google Scholar using the following terms: (brain injury OR hypoxia-ischemia OR hypoxia OR ischemia OR cerebral ischemia OR occlusion) AND (neuroprotection) AND (fetal OR neonatal OR postnatal). Other sources used to identify studies included relevant papers and reviews.

Abstracts were initially identified and screened by an investigator from our study group (R.G.) and duplicated by another investigator (J.M.D.). Full-text articles were then obtained using Papers software (version 3.4.6; Labtiva Inc., Cambridge, MA, USA) for review. Relevant studies were selected based on group consensus. Studies were deemed eligible if they presented clear histological and/or functional outcomes from in vivo experiments that investigated neuroprotection using pharmacological interventions or mechanisms of protection via less pragmatic interventions, such as gene deletion, gene inactivation, or conditioning. All pharmacological interventions were considered individually, even in studies that used more than 1 treatment. The class of drug was searched for to assess whether the neuroprotectant had potential to affect thermoregulation. Specifically, the neuroprotectants were searched for on PubMed, MEDLINE (OvidSP), and Google Scholar using the following terms: (name of relevant drug) AND (temperature OR vasodilation). The drug was considered likely to affect temperature if it had previously been shown to affect thermoregulation or belonged to a family of drugs known to affect body or brain temperature. It was deemed unlikely to affect temperature if no effect had previously been reported. Unknown indicates no studies have investigated whether the drug affects temperature. Histological outcomes were based on assessment of gray and/or white matter cell survival or death. Functional outcomes were based on assessment of behavior or recovery of brain activity, as assessed using neurophysiology or examination of clinical seizures.

Inclusion criteria were in vivo studies of rodents on postnatal days $(\mathrm{P}) 7-11$, sheep at $0.80-0.85$ of gestation and P1-5 neonatal piglets. These developmental ages are often used to study treatments for term infants, although the cortical maturity of laboratory rats at P7 is consistent with the late preterm human infant [18]. We excluded in vitro studies and those that did not meet the age criteria. The methodological quality of the studies was assessed according to whether temperature was controlled, treatment was randomized, and investigators were blinded to the intervention during histological and functional assessments. The results are summarized in Table 1 and Figures 1-4.
8

Dev Neurosci 2017;39:7-22

DOI: $10.1159 / 000452859$
Galinsky/Dean/Lear/Davidson/Dhillon/ Wassink/Bennet/Gunn 
Table 1. Preclinical studies of perinatal neuroprotection

\begin{tabular}{|c|c|c|c|c|c|c|c|c|c|}
\hline $\begin{array}{l}\text { Refer- } \\
\text { ence }\end{array}$ & Species & Insult & Treatment (route) & Timing & Temperature, ${ }^{\circ} \mathrm{C}$ & Pathology & $\begin{array}{l}\text { Functional } \\
\text { outcome }\end{array}$ & Survival & Sex \\
\hline \multicolumn{10}{|c|}{ Improved neurodevelopmental outcomes } \\
\hline 39 & $\begin{array}{l}\text { P7 } \\
\text { mice }\end{array}$ & $\begin{array}{l}\mathrm{HI}: 90 \mathrm{~min} \\
8 \% \mathrm{O}_{2}\end{array}$ & $\begin{array}{l}\text { Hydrogen (i.p.) } \\
\text { HI + vehicle } \\
\text { HI + hydrogen-rich saline }\end{array}$ & $\begin{array}{l}\text { Post-Tx: } \\
24,48,72 \mathrm{~h}\end{array}$ & $\begin{array}{l}\text { Ambient: } \\
36^{\circ} \mathrm{C} \text {, during } 2 \mathrm{~h} \\
\text { recovery }\end{array}$ & $\begin{array}{l}\downarrow \text { infarct vol, } \\
p<0.05 \text { vs. vehicle }\end{array}$ & & 3 days & ? \\
\hline 40 & $\begin{array}{l}\mathrm{P} 1 \\
\text { piglets }\end{array}$ & $\begin{array}{l}\mathrm{HI} \mathrm{FiO}_{2} \\
6 \%\end{array}$ & $\begin{array}{l}\mathrm{HT} \text { and argon } \\
\mathrm{HI}+\mathrm{HT} \\
\mathrm{HI}+\mathrm{HT}+\text { argon }\end{array}$ & $\begin{array}{l}\text { Post-Tx: } \\
2 \mathrm{~h}\end{array}$ & $\begin{array}{l}\text { Core: } \\
33.5^{\circ} \mathrm{C} \\
\text { continuous } \\
\text { monitoring }\end{array}$ & $\begin{array}{l}\sim 67 \% \downarrow \text { cell death, } \\
p<0.05 \text { vs. HT }\end{array}$ & $\begin{array}{l}\uparrow \mathrm{PCr} / \mathrm{Pi} \text { and } \downarrow \text { thalamic, } \\
\text { white matter Lac/NAA } \\
\text { and improved aEEG } \\
\text { recovery vs. HT, } p<0.05\end{array}$ & 2 days & $0^{x}$ \\
\hline 41 & $\begin{array}{l}\mathrm{P} 1-2 \\
\text { piglets }\end{array}$ & $\begin{array}{l}\mathrm{HI} \mathrm{FiO} 2 \\
10 \%\end{array}$ & $\begin{array}{l}\text { Cannabidiol (i.p.) and HT } \\
\mathrm{HI}+\mathrm{HT}+\text { vehicle } \\
\mathrm{HI}+\mathrm{HT}+\text { cannabidiol }\end{array}$ & $\begin{array}{l}\text { Post-Tx: } \\
30 \mathrm{~min}\end{array}$ & $\begin{array}{l}\text { Core: } \\
33-34^{\circ} \mathrm{C}, \\
\text { continuous } \\
\text { monitoring }\end{array}$ & $\begin{array}{l}\downarrow \text { cortical neuronal } \\
\text { necrosis vs. HT, } \\
p<0.05\end{array}$ & $\begin{array}{l}\uparrow \mathrm{Lac} / \mathrm{NAA} \text { and improved } \\
\text { aEEG recovery vs. HT, } \\
p<0.05\end{array}$ & $6 \mathrm{~h}$ & $0^{\prime}$ \\
\hline 42 & $\begin{array}{l}\mathrm{P} 7 \\
\text { rats }\end{array}$ & $\begin{array}{l}\text { HI: } 90 \mathrm{~min} \\
8 \% \mathrm{O}_{2}\end{array}$ & $\begin{array}{l}\text { Sevoflurane } \\
\mathrm{HI} \\
\mathrm{HI}+\text { sevoflurane }\end{array}$ & $\begin{array}{l}\text { Immediate } \\
\text { Tx: } 0 \mathrm{~h}\end{array}$ & $\begin{array}{l}\text { Ambient: } \\
36.5^{\circ} \mathrm{C} \text {, during } \mathrm{HI}\end{array}$ & $\begin{array}{l}\downarrow \text { CA1 and } 3 \text { hippo- } \\
\text { campal neuronal loss, } \\
p<0.05 \text { vs. HI }\end{array}$ & $\begin{array}{l}\text { Improved learning and } \\
\text { memory, } \\
p<0.05 \text { vs. HI }\end{array}$ & 3 weeks & $\sigma^{x} / 9$ \\
\hline 43 & $\begin{array}{l}\mathrm{P} 7 \\
\text { rats }\end{array}$ & $\begin{array}{l}\text { HI: } 90 \text { min } \\
8 \% \mathrm{O}_{2}\end{array}$ & $\begin{array}{l}\text { Helium } \\
\mathrm{HI} \\
\mathrm{He}+\mathrm{HI}\end{array}$ & $\begin{array}{l}\text { Pre-Tx: } \\
-24 \mathrm{~h}\end{array}$ & $\begin{array}{l}\text { Ambient: } \\
37^{\circ} \mathrm{C} \text {, during } \mathrm{HI}\end{array}$ & $\begin{array}{l}\downarrow \text { infarct vol vs. HI, } \\
p<0.05\end{array}$ & $\begin{array}{l}\text { Improved sensorimotor } \\
\text { function vs. HI }\end{array}$ & $\begin{array}{l}\text { Pathology: } \\
1 \text { day } \\
\text { behavior: } \\
3 \text { weeks }\end{array}$ & $?$ \\
\hline 44 & $\begin{array}{l}\mathrm{P} 10 \\
\text { rats }\end{array}$ & $\begin{array}{l}\text { HI: } 3.5 \mathrm{~h} \\
8 \% \mathrm{O}_{2}\end{array}$ & $\begin{array}{l}\text { MicroRNA-210 } \\
\text { antagonist (i.c.v.) } \\
\text { vehicle + HI } \\
\text { miRNA antagonist + HI }\end{array}$ & $\begin{array}{l}\text { Post-Tx: } \\
4 \mathrm{~h}\end{array}$ & $\begin{array}{l}\text { Ambient: } \\
37^{\circ} \mathrm{C} \text {, during HI }\end{array}$ & $\begin{array}{l}47 \% \downarrow \text { infarct vol vs. } \\
\text { vehicle, } p<0.05\end{array}$ & $\begin{array}{l}\text { Improved spatial learning } \\
\text { and sensorimotor } \\
\text { function vs. vehicle }\end{array}$ & $\begin{array}{l}\text { Pathology: } \\
2 \text { days } \\
\text { behavior: } \\
6 \text { weeks }\end{array}$ & $?$ \\
\hline$\overline{45}$ & $\begin{array}{l}\mathrm{P} 10 \\
\text { mice }\end{array}$ & $\begin{array}{l}\text { HI: } 15 \text { min } \\
8 \% \mathrm{O}_{2}\end{array}$ & $\begin{array}{l}\text { DHA (i.p.) } \\
\mathrm{HI}+\text { vehicle } \\
\mathrm{HI}+\text { DHA }\end{array}$ & $\begin{array}{l}\text { Immediate } \\
\text { Tx: } 0,1 \mathrm{~h}\end{array}$ & $\begin{array}{l}\text { Ambient: } \\
37^{\circ} \mathrm{C} \text {, during } \mathrm{HI}\end{array}$ & $\begin{array}{l}49 \% \downarrow \text { in infarct vol, } \\
p<0.05 \text { vs. vehicle }\end{array}$ & & 1 day & $?$ \\
\hline 45 & $\begin{array}{l}\mathrm{P} 10 \\
\text { mice }\end{array}$ & $\begin{array}{l}\mathrm{HI}: 15 \mathrm{~min} \\
8 \% \mathrm{O}_{2}\end{array}$ & $\begin{array}{l}\mathrm{HI}+\text { vehicle } \\
\mathrm{HI}+\mathrm{DHA}\end{array}$ & $\begin{array}{l}\text { Immediate } \\
\text { Tx: } 0,1 \mathrm{~h}\end{array}$ & $\begin{array}{l}\text { Ambient: } \\
37^{\circ} \mathrm{C} \text {, during } \mathrm{HI}\end{array}$ & $\begin{array}{l}32 \% \uparrow \text { in brain tissue vol, } \\
p<0.05 \text { vs. vehicle }\end{array}$ & $\begin{array}{l}\text { Improved memory } \\
\text { performance, } \\
p<0.05 \text { vs. vehicle }\end{array}$ & 9 weeks & $?$ \\
\hline 46 & $\begin{array}{l}\mathrm{P} 7 \\
\text { rats }\end{array}$ & $\begin{array}{l}\mathrm{HI}: 2 \mathrm{~h} \\
8 \% \mathrm{O}_{2}\end{array}$ & $\begin{array}{l}\text { HT and } \\
\text { NAC (i.p.) } \\
\mathrm{HI}+\mathrm{HT}+\text { vehicle } \\
\mathrm{HI}+\mathrm{HT}+\mathrm{NAC}\end{array}$ & $\begin{array}{l}\text { Immediate } \\
\text { Tx: } 0 \mathrm{~h}\end{array}$ & $\begin{array}{l}\text { Core: } 36.5-37.5 \\
\text { vs. } 33.5-34.5^{\circ} \mathrm{C} \\
\text { during } 2 \mathrm{~h} \\
\text { recovery }\end{array}$ & $\begin{array}{l}\downarrow \text { infarct vol in } \\
\text { females, } p<0.05 \\
\text { HT + NAC vs. HT } \\
\text { no effect in males }\end{array}$ & $\begin{array}{l}\text { Improved sensorimotor } \\
\text { function vs. HT. }\end{array}$ & $\begin{array}{l}\text { Pathology: } \\
2 \text { days } \\
\text { behavior: } \\
6 \text { weeks }\end{array}$ & $\sigma^{2} / 9$ \\
\hline$\overline{47}$ & $\begin{array}{l}\mathrm{P9} \\
\text { mice }\end{array}$ & $\begin{array}{l}\mathrm{HI}: 1 \mathrm{~h} \\
10 \% \mathrm{O}_{2}\end{array}$ & $\begin{array}{l}H T \\
\mathrm{HI}+\mathrm{NT} \\
\mathrm{HI}+\mathrm{HT}\end{array}$ & $\begin{array}{l}\text { Immediate } \\
\text { Tx: } 0 \mathrm{~h}\end{array}$ & $\begin{array}{l}\text { Core: } 35 \text { vs. } 27^{\circ} \mathrm{C} \\
\text { during } 4 \mathrm{~h} \\
\text { recovery }\end{array}$ & $\begin{array}{l}\uparrow \text { hippocampal } \\
\text { neuronal density } \\
\text { and } \downarrow \text { total neural } \\
\text { injury score }\end{array}$ & $\begin{array}{l}\text { Improved sensorimotor } \\
\text { function and cognition } \\
\text { vs. NT }\end{array}$ & $\begin{array}{l}\text { Pathology: } \\
1 \text { week } \\
\text { behavior: } \\
3 \text { weeks }\end{array}$ & $\sigma^{2} / 9$ \\
\hline 47 & $\begin{array}{l}\mathrm{P9} \\
\text { mice }\end{array}$ & $\begin{array}{l}\mathrm{HI}: 1 \mathrm{~h} \\
10 \% \mathrm{O}_{2}\end{array}$ & $\begin{array}{l}\mathrm{HI}+\mathrm{NT} \\
\mathrm{HI}+\mathrm{HT}\end{array}$ & $\begin{array}{l}\text { Post-Tx: } \\
2 \mathrm{~h}\end{array}$ & $\begin{array}{l}\text { Core: } 35 \text { vs. } 27^{\circ} \mathrm{C} \\
\text { during } 4 \mathrm{~h} \\
\text { recovery }\end{array}$ & $\begin{array}{l}\uparrow \text { hippocampal } \\
\text { neuronal density } \\
\text { and } \downarrow \text { neural injury } \\
\text { score }\end{array}$ & No effect on behavior & $\begin{array}{l}\text { Pathology: } \\
1 \text { week } \\
\text { behavior: } \\
5 \text { weeks }\end{array}$ & $0^{x} / 9$ \\
\hline$\overline{48}$ & $\begin{array}{l}\mathrm{P} 7 \\
\text { rats }\end{array}$ & $\begin{array}{l}\text { HI: } 2 \mathrm{~h} \\
8 \% \mathrm{O}_{2}\end{array}$ & $\begin{array}{l}\text { Dexmedetomidine } \\
\text { (i.p.) } \\
\mathrm{HI}+\text { vehicle } \\
\mathrm{HI}+\text { dexmedetomidine }\end{array}$ & $\begin{array}{l}\text { Post-Tx: } \\
30,60 \text {, and } \\
120 \mathrm{~min}\end{array}$ & $\begin{array}{l}\text { Ambient: } \\
37^{\circ} \mathrm{C} \text {, during } \mathrm{HI}\end{array}$ & & $\begin{array}{l}\text { Improved sensorimotor } \\
\text { function and learning and } \\
\text { memory vs. vehicle }\end{array}$ & 4 weeks & $?$ \\
\hline 49 & $\begin{array}{l}\text { P9 } \\
\text { mice }\end{array}$ & $\begin{array}{l}\text { HI: } 50 \mathrm{~min} \\
10 \% \mathrm{O}_{2}\end{array}$ & $\begin{array}{l}\text { Atg7 (autophagy- } \\
\text { related protein } 7 \text { ) } \\
\mathrm{WT}+\mathrm{HI} \\
\text { Atg7 } \mathrm{KO}+\mathrm{HI}\end{array}$ & $\begin{array}{l}\text { Constitutive } \\
\text { gene } \mathrm{KO}\end{array}$ & $\begin{array}{l}\text { Ambient: } \\
36^{\circ} \mathrm{C} \text {, during } \mathrm{HI}\end{array}$ & $\begin{array}{l}\sim 49 \% \downarrow \text { in infarct vol, } \\
p<0.05 \text { vs. WT }\end{array}$ & & 8 days & $\sigma^{2} / 9$ \\
\hline 50 & $\begin{array}{l}\mathrm{P7} \\
\text { rats }\end{array}$ & $\begin{array}{l}\text { HI: } 2 \mathrm{~h} \\
8 \% \mathrm{O}_{2}\end{array}$ & $\begin{array}{l}\text { Isoflurane } \\
\mathrm{HI} \\
\mathrm{HI}+\text { isoflurane }\end{array}$ & $\begin{array}{l}\text { Immediate } \\
\text { Tx: } 0 \mathrm{~h}\end{array}$ & $\begin{array}{l}\text { Ambient: } \\
37^{\circ} \mathrm{C} \text {, during } \mathrm{HI}\end{array}$ & $\begin{array}{l}\uparrow \text { cortical and } \\
\text { thalamic neuronal } \\
\text { density vs. HI }\end{array}$ & $\begin{array}{l}\text { Improved learning and } \\
\text { memory performance } \\
\text { vs. HI }\end{array}$ & $\begin{array}{l}\text { Pathology: } \\
1 \text { week } \\
\text { behavior: } \\
5 \text { weeks }\end{array}$ & $\sigma^{2} / 9$ \\
\hline 50 & $\begin{array}{l}\text { P7 } \\
\text { rats }\end{array}$ & $\begin{array}{l}\mathrm{HI}: 2 \mathrm{~h} \\
8 \% \mathrm{O}_{2}\end{array}$ & $\begin{array}{l}\mathrm{HI} \\
\mathrm{HI}+\text { isoflurane }\end{array}$ & $\begin{array}{l}\text { Post-Tx: } \\
3 \text { or } 6 h\end{array}$ & $\begin{array}{l}\text { Ambient: } \\
37^{\circ} \mathrm{C} \text {, during } \mathrm{HI}\end{array}$ & $\begin{array}{l}\uparrow \text { cortical and } \\
\text { thalamic neuronal } \\
\text { density vs. HI }\end{array}$ & $\begin{array}{l}\text { Improved learning and } \\
\text { memory performance } \\
\text { vs. HI }\end{array}$ & $\begin{array}{l}\text { Pathology: } \\
1 \text { week } \\
\text { behavior: } \\
5 \text { weeks }\end{array}$ & $\sigma^{x} / 9$ \\
\hline 51 & $\begin{array}{l}\text { P7 } \\
\text { rats }\end{array}$ & $\begin{array}{l}\text { HI: } 90 \mathrm{~min} \\
8 \% \mathrm{O}_{2}\end{array}$ & $\begin{array}{l}\text { Argon } \\
\mathrm{HI}+\text { nitrogen with } \mathrm{O}_{2} \\
\mathrm{HI}+\text { argon with } \mathrm{O}_{2}\end{array}$ & $\begin{array}{l}\text { Immediate } \\
\text { Tx: } 0 \mathrm{~h}\end{array}$ & Not specified & $\begin{array}{l}47 \% \downarrow \text { in infarct vol, } \\
p<0.05 \text { vs. nitrogen }\end{array}$ & & 4 weeks & $?$ \\
\hline 52 & $\begin{array}{l}\text { P7 } \\
\text { rats }\end{array}$ & $\begin{array}{l}\text { HI: } 2 \mathrm{~h} \\
8 \% \mathrm{O}_{2}\end{array}$ & $\begin{array}{l}\text { Taurine (i.p.) } \\
\mathrm{HI}+\text { vehicle } \\
\mathrm{HI}+\text { taurine }\end{array}$ & $\begin{array}{l}\text { Post-Tx: } \\
12,24,26, \\
48 \mathrm{~h}\end{array}$ & $\begin{array}{l}\text { Ambient: } \\
37^{\circ} \mathrm{C} \text {, during } \mathrm{HI}\end{array}$ & $\begin{array}{l}\downarrow \text { infarct vol and cell } \\
\text { death score }\end{array}$ & & 2 days & $\sigma^{x} / 9$ \\
\hline 53 & $\begin{array}{l}\mathrm{P} 10 \\
\text { mice }\end{array}$ & $\begin{array}{l}\mathrm{HI}: 25 \mathrm{~min} \\
8 \% \mathrm{O}_{2}\end{array}$ & $\begin{array}{l}\text { Creatine } \\
\text { (dietary suppl.) } \\
\mathrm{HI}+\text { normal diet } \\
\mathrm{HI}+3 \% \text { creatine }\end{array}$ & $\begin{array}{l}\text { Post-Tx: } \\
10-70 \text { days }\end{array}$ & $\begin{array}{l}\text { Core: } \\
36^{\circ} \mathrm{C} \text {, during } \mathrm{HI}\end{array}$ & $\begin{array}{l}\downarrow \text { infarct vol in creatine: } \\
17.5 \pm 2.5 \% \text { vs. normal } \\
\text { diet: } 27.8 \pm 4.1 \%, p<0.05\end{array}$ & $\begin{array}{l}\text { Improved memory and } \\
\text { motor function }\end{array}$ & 10 weeks & q \\
\hline 54 & $\begin{array}{l}\text { P1 } \\
\text { piglets }\end{array}$ & $\begin{array}{l}\mathrm{HI} \mathrm{FiO}{ }_{2} \\
12 \%\end{array}$ & $\begin{array}{l}H T \\
\mathrm{HI}+\mathrm{NT}\left(38.5^{\circ} \mathrm{C}\right) \\
\mathrm{HI}+\mathrm{HT}\left(35^{\circ} \mathrm{C}\right) \\
\mathrm{HI}+\mathrm{HT}\left(33.5^{\circ} \mathrm{C}\right) \\
\mathrm{HI}+\mathrm{HT}\left(30^{\circ} \mathrm{C}\right)\end{array}$ & $\begin{array}{l}\text { Post-Tx: } \\
2 \mathrm{~h}\end{array}$ & $\begin{array}{l}\text { Core: } \\
38.5 \text { vs. } 35,33.5 \text {, } \\
\text { or } 30^{\circ} \mathrm{C}, \\
\text { continuous } \\
\text { monitoring }\end{array}$ & $\begin{array}{l}\downarrow \text { white matter } \\
\text { apoptosis in } 35 \text { and } \\
33.5^{\circ} \mathrm{C} \text { groups } \\
\text { vs. NT, } \\
p<0.05\end{array}$ & None & 2 days & $0^{\prime}$ \\
\hline
\end{tabular}

Temperature Control and Neuroprotection
Dev Neurosci 2017;39:7-22

DOI: $10.1159 / 000452859$ 
Table 1 (continued)

\begin{tabular}{|c|c|c|c|c|c|c|c|c|c|}
\hline $\begin{array}{l}\text { Refer- } \\
\text { ence }\end{array}$ & Species & Insult & Treatment (route) & Timing & Temperature, ${ }^{\circ} \mathrm{C}$ & Pathology & $\begin{array}{l}\text { Functional } \\
\text { outcome }\end{array}$ & Survival & Sex \\
\hline 55 & $\begin{array}{l}\text { P7 } \\
\text { rats }\end{array}$ & $\begin{array}{l}\mathrm{HI}: 2 \mathrm{~h} \\
15 \mathrm{~min} \\
8 \% \mathrm{O}_{2}\end{array}$ & $\begin{array}{l}\text { Resveratrol (i.p.) } \\
\text { vehicle + HI } \\
\text { resveratrol + HI }\end{array}$ & $\begin{array}{l}\text { Pre-Tx: } \\
-10 \mathrm{~min}\end{array}$ & $\begin{array}{l}\text { Ambient: } \\
36^{\circ} \mathrm{C} \text {, during } \mathrm{HI}\end{array}$ & $\begin{array}{l}\sim 83 \% \downarrow \text { infarct vol, } \downarrow \\
\text { cortical and hippocampal } \\
\text { injury scores and } \uparrow \\
\text { myelination vs. vehicle, } \\
p<0.05\end{array}$ & $\begin{array}{l}\text { Improved memory } \\
\text { performance }\end{array}$ & $\begin{array}{l}\text { Pathology: } \\
1 \text { week } \\
\text { behavior: } \\
12 \text { weeks }\end{array}$ & $\sigma^{r} / 9$ \\
\hline 31 & $\begin{array}{l}\mathrm{P} 10 \\
\text { rats }\end{array}$ & $\begin{array}{l}\text { HI: } 45 \text { min } \\
8 \% \mathrm{O}_{2}\end{array}$ & $\begin{array}{l}H T \\
\mathrm{HI}+\mathrm{NT} \\
\mathrm{HI}+4 \mathrm{~h} \mathrm{HT}\end{array}$ & $\begin{array}{l}\text { Immediate } \\
\text { Tx: } 0 \mathrm{~h}\end{array}$ & $\begin{array}{l}\text { Core: } 31 \text { vs. } \\
36^{\circ} \mathrm{C} \text { during } 4 \mathrm{~h} \\
\text { recovery }\end{array}$ & $\begin{array}{l}\text { Greater } \downarrow \text { in regional } \\
\text { injury in males vs. females } \\
p<0.05\end{array}$ & None & $\begin{array}{l}1 \text { week } \\
\text { (8 days) }\end{array}$ & $\sigma^{\prime} / 9$ \\
\hline 31 & $\begin{array}{l}\text { P10 } \\
\text { rats }\end{array}$ & $\begin{array}{l}\text { HI: } 45 \mathrm{~min} \\
8 \% \mathrm{O}_{2}\end{array}$ & $\begin{array}{l}\mathrm{HI}+\mathrm{NT} \\
\mathrm{HI}+4 \mathrm{~h} \mathrm{HT}\end{array}$ & $\begin{array}{l}\text { Immediate } \\
\text { Tx: } 0 \mathrm{~h}\end{array}$ & $\begin{array}{l}\text { Core: } \\
31 \text { vs. } 36^{\circ} \mathrm{C} \\
\text { during } 4 \mathrm{~h} \\
\text { recovery }\end{array}$ & $\begin{array}{l}\downarrow \text { cortical and } \\
\text { hippocampal injury in } \\
\text { males; no significant } \\
\text { effect of HT in females }\end{array}$ & $\begin{array}{l}\text { HT improved working } \\
\text { memory in males but } \\
\text { not females }\end{array}$ & $\begin{array}{l}\text { Pathology } \\
\text { and } \\
\text { behavior } \\
3 \text { weeks } \\
\text { (20 days) } \\
\end{array}$ & $\sigma^{2} / 9$ \\
\hline 56 & $\begin{array}{l}\mathrm{P} 10 \\
\text { rats }\end{array}$ & $\begin{array}{l}\mathrm{HI}: 2.5 \mathrm{~h} \\
8 \% \mathrm{O}_{2}\end{array}$ & $\begin{array}{l}\text { G-CSF } \\
\text { (s.c.) } \\
\text { HI + vehicle } \\
\text { HI + G-CSF }\end{array}$ & $\begin{array}{l}\text { Post-Tx } \\
1 \mathrm{~h}\end{array}$ & $\begin{array}{l}\text { Ambient: } \\
37^{\circ} \mathrm{C} \text {, during } \mathrm{HI}\end{array}$ & $\begin{array}{l}34 \% \downarrow \text { infarct vol } \\
\text { vs. vehicle, } p<0.05\end{array}$ & None & 1 day & $?$ \\
\hline 57 & $\begin{array}{l}\text { P7 } \\
\text { mice }\end{array}$ & $\begin{array}{l}\mathrm{HI}: 100 \mathrm{~min} \\
7.5 \% \mathrm{O}_{2}\end{array}$ & $\begin{array}{l}\text { Carvacrol (i.p) } \\
\text { vehicle }+\mathrm{HI} \\
\text { carvacrol }+\mathrm{HI} \\
(30 \text { or } 50 \mathrm{mg} / \mathrm{kg})\end{array}$ & $\begin{array}{l}\text { Pre-Tx: } \\
-30 \mathrm{~min}\end{array}$ & $\begin{array}{l}\text { Ambient: } \\
37^{\circ} \mathrm{C} \text {, during } \mathrm{HI}\end{array}$ & $\begin{array}{l}\downarrow \text { infarct vol by } 46 \\
\text { and } 90 \% \text { in } 30 \text { - and } \\
50 \text {-mg groups, } \\
\text { respectively vs. vehicle, } \\
p<0.05\end{array}$ & $\begin{array}{l}\text { Improved sensorimotor } \\
\text { performance in } 50 \mathrm{mg} / \mathrm{kg} \\
\text { vs vehicle, } p<0.05\end{array}$ & $\begin{array}{l}\text { Pathology: } \\
1 \text { day } \\
\text { behavior: } \\
1 \text { week }\end{array}$ & $?$ \\
\hline 58 & $\begin{array}{l}\text { P10 } \\
\text { mice }\end{array}$ & $\begin{array}{l}\text { HI: } 25 \mathrm{~min} \\
8 \% \mathrm{O}_{2}\end{array}$ & $\begin{array}{l}\text { Creatine monohydrate } \\
\text { (dietary suppl.) } \\
\mathrm{HI}+\text { normal diet } \\
\mathrm{HI}+2 \% \text { creatine }\end{array}$ & $\begin{array}{l}\text { Post-Tx: } \\
10-105 \text { days }\end{array}$ & $\begin{array}{l}\text { Ambient: } \\
36^{\circ} \mathrm{C} \text {, during } \mathrm{HI}\end{array}$ & $\begin{array}{l}\text { No change in infarct } \\
\text { vol vs. normal diet, } \\
p<0.05\end{array}$ & $\begin{array}{l}\text { Improved motor } \\
\text { function, learning and } \\
\text { memory vs. normal diet }\end{array}$ & $\begin{array}{l}\text { Pathology: } \\
15 \text { weeks } \\
\text { behavior: } \\
17 \text { weeks } \\
\text { (118 days) }\end{array}$ & $\sigma^{x}$ \\
\hline 59 & $\begin{array}{l}\text { P7 } \\
\text { rats }\end{array}$ & $\begin{array}{l}\mathrm{HI}: 2.5 \mathrm{~h} \\
8 \% \mathrm{O}_{2}\end{array}$ & $\begin{array}{l}\text { Uridine (i.p.) } \\
\mathrm{HI}+\text { vehicle } \\
\mathrm{HI}+\text { uridine }\end{array}$ & $\begin{array}{l}\text { Immediate } \\
\text { Tx: } 0 \text { h, } 1 \text {, } \\
\text { and } 2 \text { days }\end{array}$ & $\begin{array}{l}\text { Ambient: } \\
37^{\circ} \mathrm{C} \text {, during } \mathrm{HI}\end{array}$ & $\begin{array}{l}\downarrow \text { infarct vol: uridine, } \\
11 \pm 1 \% \text { vs. vehicle, } \\
20 \pm 3 \%, p<0.05\end{array}$ & None & 3 days & $\sigma^{x} / 9$ \\
\hline 60 & $\begin{array}{l}\text { P10 } \\
\text { mice }\end{array}$ & $\begin{array}{l}\mathrm{HI}: 45 \mathrm{~min} \\
8 \% \mathrm{O}_{2}\end{array}$ & $\begin{array}{l}H T \\
\mathrm{HI}+\mathrm{NT} \\
\mathrm{HI}+4 \mathrm{hHT}\end{array}$ & $\begin{array}{l}\text { Immediate } \\
\text { Tx: } 0 \mathrm{~h}\end{array}$ & $\begin{array}{l}\text { Ambient: } \\
37 \text { vs. } 28.5^{\circ} \mathrm{C} \\
\text { during } 4 \text { h of } \\
\text { recovery }\end{array}$ & $\begin{array}{l}\sim 34 \% \uparrow \mathrm{NeuN}+\text { staining } \\
\text { area vs. HI }+\mathrm{NT}, \\
p<0.05\end{array}$ & None & 1 week & $\sigma^{x}$ \\
\hline 61 & $\begin{array}{l}\text { P7 } \\
\text { rats }\end{array}$ & $\begin{array}{l}\mathrm{HI}: 2.5 \mathrm{~h} \\
8 \% \mathrm{O}_{2}\end{array}$ & $\begin{array}{l}\text { Gap26 (Cx43 mimetic } \\
\text { peptide) (i.p.) } \\
\text { vehicle + HI } \\
\text { Gap26 + HI }\end{array}$ & $\begin{array}{l}\text { Pre-Tx: } \\
-1 \mathrm{~h}\end{array}$ & $\begin{array}{l}\text { Ambient: } \\
37^{\circ} \mathrm{C} \text {, during } \mathrm{HI}\end{array}$ & $\begin{array}{l}\downarrow \text { infarct vol by } \sim 60 \% \text { vs. } \\
\text { vehicle, } p<0.05\end{array}$ & & 2 days & $?$ \\
\hline 61 & $\begin{array}{l}\text { P7 } \\
\text { rats }\end{array}$ & $\begin{array}{l}\mathrm{HI}: 2.5 \mathrm{~h} \\
8 \% \mathrm{O}_{2}\end{array}$ & $\begin{array}{l}\text { Gap26 (Cx43 } \\
\text { mimetic peptide) (i.p.) } \\
\mathrm{HI}+\text { vehicle } \\
\mathrm{HI}+\text { Gap26 }\end{array}$ & $\begin{array}{l}\text { Post-Tx: } \\
24 \mathrm{~h} \text { then } \\
\text { daily for } 7 \text { days }\end{array}$ & $\begin{array}{l}\text { Ambient: } \\
37^{\circ} \mathrm{C} \text {, during } \mathrm{HI}\end{array}$ & None & $\begin{array}{l}\text { Improved motor function } \\
\text { and memory vs. vehicle }\end{array}$ & 3 weeks & $?$ \\
\hline 62 & $\begin{array}{l}\text { P7 } \\
\text { rats }\end{array}$ & $\begin{array}{l}\mathrm{HI}: 90 \mathrm{~min} \\
8 \% \mathrm{O}_{2}\end{array}$ & $\begin{array}{l}H T \text { and } X e \\
\mathrm{HI}+\mathrm{NT} \\
\mathrm{HI}+5 \mathrm{~h} \mathrm{HT} \\
\mathrm{HI}+5 \mathrm{~h} \mathrm{HT}+50 \% \mathrm{Xe}\end{array}$ & $\begin{array}{l}\text { Post-Tx: } \\
5 \mathrm{~h}\end{array}$ & $\begin{array}{l}\text { Core: } \\
36^{\circ} \mathrm{C} \text {, during } \mathrm{HI} \\
37 \text { vs. } 32^{\circ} \mathrm{C} \text {, } \\
\text { during } 5 \text { h of } \\
\text { recovery }\end{array}$ & $\begin{array}{l}\text { No additive effect } \\
\text { of Xe on infarct area } \\
\text { vs. HT, } p>0.05\end{array}$ & $\begin{array}{l}\text { HT }+ \text { Xe improved } \\
\text { sensorimotor } \\
\text { performance vs. } \\
\text { HT; negative correlation } \\
\text { between brain injury and } \\
\text { motor function females } \\
(r=-0.76, p<0.05) \text { not } \\
\text { males }(p=0.06)\end{array}$ & $\begin{array}{l}\text { Pathology: } \\
10 \text { weeks } \\
\text { behavior: } \\
9 \text { weeks }\end{array}$ & $\sigma^{\prime \prime} / 9$ \\
\hline 63 & $\begin{array}{l}\text { P7 } \\
\text { rats }\end{array}$ & $\begin{array}{l}\mathrm{HI}: 150 \mathrm{~min} \\
8 \% \mathrm{O}_{2}\end{array}$ & $\begin{array}{l}\text { Vitexin (5, 7, 4-trihydroxy- } \\
\text { flavone-8-glucoside) (i.p.) } \\
\text { HI + vehicle } \\
\text { HI + vitexin }\end{array}$ & $\begin{array}{l}\text { Post-Tx: } \\
5 \text { min }\end{array}$ & $\begin{array}{l}\text { Ambient: } \\
37^{\circ} \mathrm{C} \text {, during } \mathrm{HI}\end{array}$ & $\begin{array}{l}\sim 64 \% \downarrow \text { infarction volume } \\
\text { and } \downarrow \text { cortical and hippo- } \\
\text { campal neuronal cell } \\
\text { death, } p<0.05 \text { vs. vehicle }\end{array}$ & None & 2 days & $\sigma^{r} / 9$ \\
\hline 29 & $\begin{array}{l}\mathrm{P} 10-11 \\
\text { rats }\end{array}$ & $\begin{array}{l}\mathrm{HI}: 1 \mathrm{~h} \\
8 \% \mathrm{O}_{2}\end{array}$ & $\begin{array}{l}H T \\
\mathrm{HI}+\mathrm{NT} \\
\mathrm{HI}+4 \mathrm{hHT}\end{array}$ & $\begin{array}{l}\text { Immediate } \\
\text { Tx: } 0 \mathrm{~h}\end{array}$ & $\begin{array}{l}\text { Core: } \\
36-36.5 \text { vs. } \\
32^{\circ} \mathrm{C} \text {, during } \\
4 \text { h of } \\
\text { recovery }\end{array}$ & $\begin{array}{l}\text { At week } 12: \downarrow \text { total and } \\
\text { regional damage scores } \\
\text { vs. } \mathrm{HI}+\mathrm{NT}, p<0.05\end{array}$ & $\begin{array}{l}\text { HT improved motor } \\
\text { function in moderate but } \\
\text { not severely injured } \\
\text { subjects vs. NT, } p<0.05\end{array}$ & $\begin{array}{l}\text { Pathology: } \\
12 \text { weeks } \\
\text { behavior: } \\
9 \text { weeks }\end{array}$ & $\sigma^{r} / 9$ \\
\hline 64 & $\begin{array}{l}\text { P7 } \\
\text { rats }\end{array}$ & $\begin{array}{l}\text { HI: } 120 \mathrm{~min} \\
8 \% \mathrm{O}_{2}\end{array}$ & $\begin{array}{l}\text { NAC } \\
\text { (i.p.) } \\
\text { vehicle + HI } \\
\text { NAC + HI } \\
\end{array}$ & $\begin{array}{l}\text { Pre-Tx: } \\
-30 \text { min, then } \\
\text { daily from P8 } \\
\text { to P44 }\end{array}$ & $\begin{array}{l}\text { Ambient: } \\
36^{\circ} \mathrm{C} \text {, during } \mathrm{HI}\end{array}$ & $\begin{array}{l}\uparrow \mathrm{MBP} \text { expression in } \\
\text { corpus callosum vs. } \\
\text { vehicle, } p<0.05\end{array}$ & $\begin{array}{l}\text { Improved sensorimotor } \\
\text { function vs. vehicle }\end{array}$ & 4 weeks & $\sigma^{x}$ \\
\hline 65 & $\begin{array}{l}\text { P7 } \\
\text { rats }\end{array}$ & $\begin{array}{l}\mathrm{HI} 3.5 \mathrm{~h} \\
8 \% \mathrm{O}_{2}\end{array}$ & $\begin{array}{l}\text { Progesterone } \\
\text { (i.p. and s.c.) } \\
\text { HI + vehicle } \\
\text { HI + progesterone }\end{array}$ & $\begin{array}{l}\text { Immediate } \\
\text { Tx: } 0 \text { h i.p. } \\
\text { then } 2 \mathrm{~h} \text { and } \\
1,2,3,4,5,6 \text {, } \\
\text { and } 7 \text { days s.c. }\end{array}$ & $\begin{array}{l}\text { Ambient: } \\
37^{\circ} \mathrm{C} \text {, during } \mathrm{HI}\end{array}$ & $\begin{array}{l}30 \% \downarrow \text { mean tissue } \\
\text { loss in HI + progesterone } \\
\text { males vs. vehicle } \\
\text { (no effect in females) }\end{array}$ & $\begin{array}{l}\text { Behavioral tests showed } \\
\text { no benefit of progesterone } \\
\text { vs. vehicle, } p>0.05\end{array}$ & $\begin{array}{l}\text { Pathology: } \\
7 \text { weeks } \\
\text { behavior: } \\
6 \text { weeks }\end{array}$ & $\sigma^{\prime} / 9$ \\
\hline 66 & $\begin{array}{l}\text { P7 } \\
\text { rats }\end{array}$ & $\begin{array}{l}\text { HI: } 2 \mathrm{~h} \\
8 \% \mathrm{O}_{2}\end{array}$ & $\begin{array}{l}\text { HT } \\
\text { NT during HI } \\
\text { HT during HI }\end{array}$ & During HI & $\begin{array}{l}\text { Ambient: } \\
36.1 \text { vs. } 32.2^{\circ} \mathrm{C} \\
\text { during } 2 \mathrm{~h} \mathrm{HI} \text {, } \\
35.7-37.0^{\circ} \mathrm{C} \\
\text { during recovery } \\
\end{array}$ & None & $\begin{array}{l}\text { Greater improvement in } \\
\text { sensory motor and } \\
\text { memory performance in } \\
\text { HT females vs. HT males } \\
\text { vs. NT }\end{array}$ & $\begin{array}{l}10-11 \\
\text { weeks } \\
(73-76 \\
\text { days })\end{array}$ & $\sigma^{\prime} / 9$ \\
\hline
\end{tabular}


Table 1 (continued)

\begin{tabular}{|c|c|c|c|c|c|c|c|c|c|}
\hline $\begin{array}{l}\text { Refer- } \\
\text { ence }\end{array}$ & Species & Insult & Treatment (route) & Timing & Temperature, ${ }^{\circ} \mathrm{C}$ & Pathology & $\begin{array}{l}\text { Functional } \\
\text { outcome }\end{array}$ & Survival & Sex \\
\hline 67 & $\begin{array}{l}\text { P10 } \\
\text { mice }\end{array}$ & $\begin{array}{l}\mathrm{HI}: 15 \mathrm{~min} \\
8 \% \mathrm{O}_{2}\end{array}$ & $\begin{array}{l}\text { Isoflurane } \\
\mathrm{HI}+\text { room air } \\
\mathrm{HI}+\text { isoflurane }\end{array}$ & $\begin{array}{l}\text { Immediate } \\
\text { Tx: } 0 \mathrm{~h}\end{array}$ & $\begin{array}{l}\text { Ambient: } \\
37-37.5^{\circ} \mathrm{C} \text {, } \\
\text { during } \mathrm{HI}\end{array}$ & $\begin{array}{l}37 \% \downarrow \text { infarct vol } \\
\text { vs. room air }\end{array}$ & $\begin{array}{l}\text { Improved memory } \\
\text { performance vs. room air }\end{array}$ & $\begin{array}{l}\text { Pathology: } \\
1 \text { day } \\
\text { behavior: } \\
8 \text { weeks }\end{array}$ & $?$ \\
\hline 68 & $\begin{array}{l}\text { P7-9 } \\
\text { mice }\end{array}$ & $\begin{array}{l}\mathrm{HI}: 60 \mathrm{~min} \\
7.5 \% \mathrm{O}_{2}\end{array}$ & $\begin{array}{l}\text { HPC and } K_{\mathrm{ATP}} \text { channel } \\
\text { blocker (tolbutamide) } \\
\text { (i.p.) } \\
\text { HI } \\
\text { HPC + tolbutamide + HI } \\
\text { HPC + vehicle + HI }\end{array}$ & $\begin{array}{l}\text { Pre-Tx: } \\
-2 \text { days }\end{array}$ & $\begin{array}{l}\text { Core: } \\
37^{\circ} \mathrm{C} \text {, during } \mathrm{HI} \\
\text { and } 30 \text { min of } \\
\text { recovery }\end{array}$ & $\begin{array}{l}\downarrow \text { infarct vol in HPC }+ \\
\text { vehicle: } 21.4 \pm 3.5 \% \text { vs. } \\
\text { HPC + tolbutamide: } 45.3 \\
\pm 1.7 \%, p<0.05 ; \text { similar } \\
\text { infarct vol between HI } \\
\text { and HPC + tolbutamide }\end{array}$ & None & 1 day & $\sigma^{2} / 9$ \\
\hline 68 & $\begin{array}{l}\text { P7-9 } \\
\text { mice }\end{array}$ & $\begin{array}{l}\text { HI: } 100 \mathrm{~min} \\
7.5 \% \mathrm{O}_{2}\end{array}$ & $\begin{array}{l}\mathrm{HI} \\
\mathrm{HPC}+\text { vehicle + HI } \\
\mathrm{HPC}+\text { tolbutamide + HI }\end{array}$ & $\begin{array}{l}\text { Pre-Tx: } \\
2 \text { days }\end{array}$ & $\begin{array}{l}\text { Core: } \\
37^{\circ} \mathrm{C} \text {, during } \mathrm{HI} \\
\text { and } 30 \text { min of } \\
\text { recovery }\end{array}$ & $\begin{array}{l}\downarrow \text { infarct vol in HPC }+ \\
\text { vehicle by } \sim 45 \text { and } 40 \% \\
\text { vs. HI and HPC }+ \\
\text { tolbutamide, respectively, } \\
p<0.05\end{array}$ & $\begin{array}{l}\text { Improved sensorimotor } \\
\text { performance in HPC vs. } \\
\text { HPC + tolbutamide, } \\
p<0.05\end{array}$ & $\begin{array}{l}\text { Pathology } \\
\text { and } \\
\text { behavior: } 1 \\
\text { week }\end{array}$ & $\sigma^{2} / 9$ \\
\hline 69 & $\begin{array}{l}\text { P7 } \\
\text { rats }\end{array}$ & $\begin{array}{l}\text { HI: } 2.5 \mathrm{~h} \\
8 \% \mathrm{O}_{2}\end{array}$ & $\begin{array}{l}H T \\
\mathrm{HI}+\mathrm{NT} \\
\mathrm{HI}+3 \mathrm{~h} \mathrm{HT}\end{array}$ & $\begin{array}{l}\text { Immediate } \\
\text { Tx: } 0 \mathrm{~h}\end{array}$ & $\begin{array}{l}\text { Core: } \\
38 \text { vs. } 30^{\circ} \mathrm{C} \text {, during } \\
3 \mathrm{~h} \text { of recovery }\end{array}$ & None & $\begin{array}{l}\text { Improved } \\
\text { neurobehavioral scores } \\
\text { vs. NT }\end{array}$ & $3 \mathrm{~h}$ & $\sigma^{\prime \prime}$ \\
\hline 70 & $\begin{array}{l}\mathrm{P} 7 \\
\text { rats }\end{array}$ & $\begin{array}{l}\mathrm{HI}: 2.5 \mathrm{~h} \\
8 \% \mathrm{O}_{2}\end{array}$ & $\begin{array}{l}\text { P5-TAT (i.p.) } \\
\text { vehicle + HI } \\
\text { P5-TAT + HI } \\
(1,10,25,50 \mu \mathrm{g} / \mathrm{kg})\end{array}$ & $\begin{array}{l}\text { Pre-Tx: } \\
-1 \mathrm{~h}\end{array}$ & $\begin{array}{l}\text { Ambient: } \\
37^{\circ} \mathrm{C} \text {, during } \mathrm{HI}\end{array}$ & $\begin{array}{l}\text { Dose-dependent } \downarrow \text { infarct } \\
\text { vol. } 58 \% \downarrow \text { in } 50 \mu \mathrm{g} / \mathrm{kg} \\
\text { group, } p<0.05\end{array}$ & None & 2 days & $?$ \\
\hline 70 & $\begin{array}{l}\text { P7 } \\
\text { rats }\end{array}$ & $\begin{array}{l}\mathrm{HI}: 2.5 \mathrm{~h} \\
8 \% \mathrm{O}_{2}\end{array}$ & $\begin{array}{l}\mathrm{HI}+\text { vehicle } \\
\mathrm{HI}+\mathrm{P} 5-\mathrm{TAT} \\
(50 \mu \mathrm{g} / \mathrm{kg} / \mathrm{day})\end{array}$ & $\begin{array}{l}\text { Post-Tx: } \\
1-7 \text { days } \\
\text { after HI }\end{array}$ & $\begin{array}{l}\text { Ambient: } \\
37^{\circ} \mathrm{C} \text {, during } \mathrm{HI}\end{array}$ & None & $\begin{array}{l}\text { Improved } \\
\text { sensorimotor } \\
\text { performance vs. vehicle }\end{array}$ & 4 weeks & $?$ \\
\hline 71 & $\begin{array}{l}\text { P8 } \\
\text { mice }\end{array}$ & $\begin{array}{l}\mathrm{HI}: 30 \mathrm{~min} \\
10 \% \mathrm{O}_{2}\end{array}$ & $\begin{array}{l}\text { NP1 } \\
\text { (neuronal pentraxin 1) } \\
\text { WT + HI } \\
\text { NP1 KO + HI }\end{array}$ & $\begin{array}{l}\text { Constitutive } \\
\text { gene } \\
\text { knockout }\end{array}$ & $\begin{array}{l}\text { Ambient: } \\
35^{\circ} \mathrm{C} \text {, during } 2 \mathrm{~h} \\
\text { recovery }\end{array}$ & $\begin{array}{l}\uparrow \text { cortical, striatal, and } \\
\text { hippocampal vol by } \sim 45 \text {, } \\
50 \text { and } 50 \% \text {, respectively, } \\
p<0.05 \text { vs. WT }\end{array}$ & None & 1 week & $?$ \\
\hline 72 & $\begin{array}{l}\text { P7 } \\
\text { rats }\end{array}$ & $\begin{array}{l}\mathrm{HI}: 2 \mathrm{~h} \\
8 \% \mathrm{O}_{2}\end{array}$ & $\begin{array}{l}\text { iNOS inhibition (i.c.v.) } \\
\text { vehicle }+\mathrm{HI} \\
\text { iNOS inhibitor + HI }\end{array}$ & $\begin{array}{l}\text { Pre-Tx: } \\
-4 \text { days }\end{array}$ & $\begin{array}{l}\text { Ambient: } \\
37^{\circ} \mathrm{C} \text {, during } \mathrm{HI}\end{array}$ & $\begin{array}{l}\uparrow \text { hippocampal neuronal } \\
\text { density by } \sim 67 \text { and } 64 \% \\
\text { in CA } 1 \text { and CA3, } \\
\text { respectively. } p<0.05 \\
\text { vs. vehicle }\end{array}$ & $\begin{array}{l}\text { Improved motor } \\
\text { function, learning and } \\
\text { memory, } \\
p<0.05 \text { vs. vehicle }\end{array}$ & $\begin{array}{l}\text { Pathology: } \\
1 \text { week } \\
\text { behavior: } \\
3 \text { weeks }\end{array}$ & $\sigma^{\prime} / 9$ \\
\hline 73 & $\begin{array}{l}\text { P7 } \\
\text { rats }\end{array}$ & $\begin{array}{l}\mathrm{HI}: 150 \mathrm{~min} \\
8 \% \mathrm{O}_{2}\end{array}$ & $\begin{array}{l}\text { OMT (oxymatrine) } \\
\text { (i.p.) } \\
\text { vehicle + HI } \\
\text { OMT + HI } \\
\end{array}$ & $\begin{array}{l}\text { Pre-Tx: } \\
-15 \text { min then } \\
\text { every } 12 \mathrm{~h} \text { for } \\
2 \text { days post-HI }\end{array}$ & $\begin{array}{l}\text { Ambient: } \\
37^{\circ} \mathrm{C} \text {, during } \mathrm{HI}\end{array}$ & $\begin{array}{l}\sim 55 \% \downarrow \text { infarct area and } \\
\downarrow \text { neuronal cell death } \\
\text { vs. vehicle, } p<0.05\end{array}$ & $\begin{array}{l}\text { Improved sensorimotor } \\
\text { function and memory vs } \\
\text { vehicle, } p<0.05\end{array}$ & $\begin{array}{l}\text { Pathology: } \\
2 \text { days } \\
\text { behavior: } \\
7 \text { weeks }\end{array}$ & $\sigma^{\prime \prime}$ \\
\hline 74 & $\begin{array}{l}\text { P9 } \\
\text { mice }\end{array}$ & $\begin{array}{l}\mathrm{LPS}+\mathrm{HI} \\
20 \mathrm{~min} \\
10 \% \mathrm{O}_{2}\end{array}$ & $\begin{array}{l}\text { IDR-1018 (innate defense } \\
\text { regulator peptide 1018) (i.p.) } \\
\text { LPS + HI + vehicle } \\
\text { LPS + HI + IDR-1018 }\end{array}$ & $\begin{array}{l}\text { Post-Tx: } \\
3 \mathrm{~h}\end{array}$ & Not specified & $\begin{array}{l}\downarrow \text { brain injury score in } \\
\text { cortex, thalamus } \\
\text { hippocampus and } \\
\text { striatum and } \downarrow \text { white } \\
\text { matter loss by } \sim 80 \% \text {, } \\
p<0.05 \text { vs. vehicle }\end{array}$ & None & 1 week & $\sigma^{\prime \prime} / 9$ \\
\hline 75 & $\begin{array}{l}\text { P7 } \\
\text { rats }\end{array}$ & $\begin{array}{l}\mathrm{HI}: 2 \mathrm{~h} \\
8 \% \mathrm{O}_{2}\end{array}$ & $\begin{array}{l}\text { Sildenafil (i.p.) } \\
\mathrm{HI}+\text { vehicle } \\
\mathrm{HI}+\text { sildenafil citrate }\end{array}$ & $\begin{array}{l}\text { Immediate } \\
\text { Tx: } 0 \mathrm{~h}\end{array}$ & Not specified & $\begin{array}{l}\downarrow \text { brain tissue loss by } 43 \pm \\
15 \% \text { and } \uparrow \text { myelination in } \\
\text { cingulum and external } \\
\text { capsule, } p<0.05 \text { vs } \\
\text { vehicle }\end{array}$ & $\begin{array}{l}\text { Intermediate } \\
\text { improvement in motor } \\
\text { performance vs. vehicle }\end{array}$ & 1 week & $\sigma^{\prime \prime} / 9$ \\
\hline 76 & $\begin{array}{l}\mathrm{P} 10 \\
\text { rats }\end{array}$ & $\begin{array}{l}\mathrm{HI}: 120 \mathrm{~min} \\
8 \% \mathrm{O}_{2}\end{array}$ & $\begin{array}{l}\text { Dexamethasone (i.c.v) } \\
\text { vehicle + HI } \\
\text { dexamethasone + HI }\end{array}$ & $\begin{array}{l}\text { Pre-Tx: } \\
\text { immediately } \\
\text { before HI }\end{array}$ & Not specified & $\begin{array}{l}\sim 57 \% \downarrow \text { infarction size } \\
\text { vs. vehicle }\end{array}$ & None & 2 days & $\sigma^{r} / 9$ \\
\hline 77 & $\begin{array}{l}\text { P9 } \\
\text { mice }\end{array}$ & $\begin{array}{l}\mathrm{HI}: 50 \mathrm{~min} \\
10 \% \mathrm{O}_{2}\end{array}$ & $\begin{array}{l}\text { Q-VD-OPh (broad-spectrum } \\
\text { caspase inhibitor) (i.p.) } \\
\text { HI + vehicle } \\
\text { HI + Q-VD-OPh }\end{array}$ & $\begin{array}{l}\text { Post-Tx: } \\
12 \mathrm{~h} \text { then every } \\
24 \mathrm{~h} \\
\text { for } 2 \text { weeks }\end{array}$ & $\begin{array}{l}\text { Ambient: } \\
36^{\circ} \mathrm{C} \text {, during } \mathrm{HI}\end{array}$ & $\begin{array}{l}\downarrow \text { tissue loss (by } 31.3 \% \text { ), } \\
\text { decreased neuropatholo- } \\
\text { gical scores in cortex, } \\
\text { hippocampus, and } \\
\text { thalamus vs. vehicle, } \\
p<0.05\end{array}$ & $\begin{array}{l}\text { Improved } \\
\text { sensorimotor } \\
\text { function vs. } \\
\text { vehicle, } \\
p<0.05\end{array}$ & $\begin{array}{l}\text { Behavior: } \\
6 \text { weeks } \\
\text { pathology: } \\
16 \text { weeks }\end{array}$ & $\sigma^{x}$ \\
\hline 78 & $\begin{array}{l}\text { P7 } \\
\text { rats }\end{array}$ & $\begin{array}{l}\mathrm{HI}: 2 \mathrm{~h} \\
8 \% \mathrm{O}_{2}\end{array}$ & $\begin{array}{l}n N O S \text { (7-nitroindazole) } \\
\text { or iNOS (aminoguanidine) } \\
\text { inhibition (i.p.) } \\
\text { vehicle + HI } \\
\text { 7-nitroindazole + HI } \\
\text { aminoguanidine + HI }\end{array}$ & $\begin{array}{l}\text { Pre-Tx: } \\
30 \text { min }\end{array}$ & Not specified & $\begin{array}{l}\text { Complete and partial } \\
\text { reduction in cortical } \\
\text { infarct vol in nNOS and } \\
\text { iNOS inhibitor groups, } \\
\text { respectively, vs. vehicle, } \\
p<0.05\end{array}$ & None & 1 week & $\sigma^{x}$ \\
\hline 78 & $\begin{array}{l}\text { P7 } \\
\text { rats }\end{array}$ & $\begin{array}{l}\mathrm{HI}: 2 \mathrm{~h} \\
8 \% \mathrm{O}_{2}\end{array}$ & $\begin{array}{l}\mathrm{HI}+\text { vehicle } \\
\mathrm{HI}+7 \text {-nitroindazole } \\
\mathrm{HI}+\text { aminoguanidine }\end{array}$ & $\begin{array}{l}\text { Post-Tx: } \\
3 \mathrm{~h}\end{array}$ & Not specified & $\begin{array}{l}\text { Partial reduction in } \\
\text { cortical infarct vol in } \\
\text { iNOS group vs. nNOS } \\
\text { and vehicle, } p<0.05\end{array}$ & None & 1 week & $\sigma^{x}$ \\
\hline 79 & $\begin{array}{l}\text { Fetal } \\
\text { sheep } \\
(0.85 \\
\text { GA })\end{array}$ & $\begin{array}{l}5 \times 10 \mathrm{~min} \\
\mathrm{UCO}\end{array}$ & $\begin{array}{l}\text { Allopurinol } \\
\text { (maternal) (i.v.) } \\
\text { UCO + vehicle } \\
\text { UCO + allopurinol }\end{array}$ & $\begin{array}{l}\text { Tx during } \\
\text { UCO }\end{array}$ & Not specified & $\begin{array}{l}\downarrow \text { hippocampal (CA3 and } \\
\text { CA4) neuronal damage } \\
\text { score vs. vehicle }\end{array}$ & None & 2 days & $?$ \\
\hline
\end{tabular}

Temperature Control and Neuroprotection
Dev Neurosci 2017;39:7-22

DOI: 10.1159/000452859 
Table 1 (continued)

\begin{tabular}{|c|c|c|c|c|c|c|c|c|c|}
\hline $\begin{array}{l}\text { Refer- } \\
\text { ence }\end{array}$ & Species & Insult & Treatment (route) & Timing & Temperature, ${ }^{\circ} \mathrm{C}$ & Pathology & $\begin{array}{l}\text { Functional } \\
\text { outcome }\end{array}$ & Survival & Sex \\
\hline 80 & $\begin{array}{l}\text { P7 } \\
\text { rats }\end{array}$ & $\begin{array}{l}\mathrm{HI}: 120 \mathrm{~min} \\
8 \% \mathrm{O}_{2}\end{array}$ & $\begin{array}{l}\text { Caffeine citrate (i.p.) } \\
\text { caffeine + HI } \\
\text { vehicle + HI }\end{array}$ & $\begin{array}{l}\text { Pre-Tx: } \\
\text { immediately } \\
\text { before HI } \\
\text { then } 0,24, \\
48 \text {, and } 72 \mathrm{~h}\end{array}$ & $\begin{array}{l}\text { Ambient: } \\
37^{\circ} \mathrm{C} \text {, during } \mathrm{HI}\end{array}$ & $\begin{array}{l}\downarrow \text { cell death in the } \\
\text { hippocampus and parietal } \\
\text { cortex vs. vehicle, } p<0.05\end{array}$ & None & 1 week & $?$ \\
\hline 81 & $\begin{array}{l}\mathrm{P} 7 \\
\text { rats }\end{array}$ & $\begin{array}{l}\mathrm{HI}: 2.5 \mathrm{~h} \\
8 \% \mathrm{O}_{2}\end{array}$ & $\begin{array}{l}29 \mathrm{D} 7 \mathrm{mAb} \text { (tropomyosin } \\
\text { related kinase B agonist) } \\
\text { (i.c.v.) } \\
\text { vehicle }+\mathrm{HI} \\
29 \mathrm{D} 7(0.1 \mathrm{nmol})+\mathrm{HI} \\
29 \mathrm{D} 7(0.3 \mathrm{nmol})+\mathrm{HI}\end{array}$ & $\begin{array}{l}\text { Pre-Tx: } \\
\text { immediately } \\
\text { before HI }\end{array}$ & $\begin{array}{l}\text { Core: } \\
35-37^{\circ} \mathrm{C}, \\
\text { measured } \\
\text { intermittently for } \\
7 \text { days post-HI }\end{array}$ & $\begin{array}{l}\downarrow \% \text { area tissue loss in } \\
\text { striatum, hippocampus } \\
\text { and cortex } \\
p<0.050 .1 \text { and } 0.3 \mathrm{nmol} \\
\text { vs. vehicle, } p<0.05\end{array}$ & None & 1 week & $\sigma^{\prime} / 9$ \\
\hline 81 & $\begin{array}{l}\mathrm{P} 7 \\
\text { rats }\end{array}$ & $\begin{array}{l}\text { HI: } 2.5 \mathrm{~h} \\
8 \% \mathrm{O}_{2}\end{array}$ & $\begin{array}{l}\text { vehicle + HI } \\
29 \mathrm{D} 7(0.3 \mathrm{nmol})+\mathrm{HI}\end{array}$ & $\begin{array}{l}\text { Pre-Tx: } \\
\text { immediately } \\
\text { before HI }\end{array}$ & $\begin{array}{l}\text { Core: } \\
35-37^{\circ} \mathrm{C}, \\
\text { measured } \\
\text { intermittently for } \\
7 \text { days post-HI }\end{array}$ & $\begin{array}{l}\downarrow \% \text { area tissue loss in } \\
\text { striatum, hippocampus } \\
\text { and cortex by } \sim 72,77 \\
\text { and } 77 \%, \text { respectively, } \\
p<0.05 \text { vs. vehicle }\end{array}$ & $\begin{array}{l}\text { Improved } \\
\text { sensory motor function } \\
\text { vs. vehicle, } p<0.05\end{array}$ & 5 weeks & $\sigma^{\pi} / 9$ \\
\hline 82 & $\begin{array}{l}\mathrm{P7} \\
\text { mice }\end{array}$ & $\begin{array}{l}\text { HI: } 30 \mathrm{~min} \\
8 \% \mathrm{O}_{2}\end{array}$ & $\begin{array}{l}\text { Cl-amidine (peptidyl- } \\
\text { arginine deiminase } \\
\text { inhibition) (i.p.) } \\
\text { LPS + HI + vehicle } \\
\text { LPS + HI + Cl-amidine }\end{array}$ & $\begin{array}{l}\text { Immediate } \\
\text { Tx: } 0 \mathrm{~h}\end{array}$ & $\begin{array}{l}\text { Ambient: } \\
36^{\circ} \mathrm{C} \text {, during } \mathrm{HI}\end{array}$ & $\begin{array}{l}\downarrow \text { infarct vol in the } \\
\text { hippocampus and } \\
\text { external capsule by } \sim 62 \\
\text { and } 74 \% \text {, respectively, } \\
p<0.05\end{array}$ & None & 2 days & $?$ \\
\hline 83 & $\begin{array}{l}\text { P10 } \\
\text { mice }\end{array}$ & $\begin{array}{l}\text { HI: } 60 \mathrm{~min} \\
10 \% \mathrm{O}_{2}\end{array}$ & $\begin{array}{l}\text { HT and sevoflurane } \\
\mathrm{HI}+10 \% \mathrm{O}_{2} 1 \mathrm{~h} \\
\mathrm{HI}+3.5 \% \text { sevoflurane }+ \\
\mathrm{HT}(1 \mathrm{~h})\end{array}$ & $\begin{array}{l}\text { Immediate } \\
\text { Tx: } 0 \mathrm{~h}\end{array}$ & $\begin{array}{l}\text { Core: } \sim 34 \text { vs. } \\
36.5-37.5^{\circ} \mathrm{C} \\
\text { during HI }\end{array}$ & $\begin{array}{l}\downarrow \text { neuronal damage score } \\
\text { in neocortex, striatum, } \\
\text { thalamus, and } \\
\text { hippocampus vs. HI }+ \\
10 \% \mathrm{O}_{2}, p<0.05\end{array}$ & $\begin{array}{l}\text { Improved learning and } \\
\text { memory vs. } \\
\mathrm{HI}+10 \% \mathrm{O}_{2} \\
p<0.05\end{array}$ & $\begin{array}{l}\text { Pathology } \\
1 \text { week } \\
\text { behavior: } \\
8 \text { weeks }\end{array}$ & $\sigma^{\prime \prime} / 9$ \\
\hline 84 & $\begin{array}{l}\mathrm{P} 7 \\
\text { rats }\end{array}$ & $\begin{array}{l}\text { HI: } 90 \text { min } \\
8 \% \mathrm{O}_{2}\end{array}$ & $\begin{array}{l}\text { HT and Xe } \\
\mathrm{HI}+\mathrm{NT} \\
\mathrm{HI}+5 \mathrm{~h} \mathrm{HT} \\
\mathrm{HI}+5 \mathrm{~h} \mathrm{HT}+\mathrm{Xe} 50 \%\end{array}$ & $\begin{array}{l}\text { Immediate } \\
\text { Tx: } 0 \mathrm{~h}\end{array}$ & $\begin{array}{l}\text { Core: } \\
36^{\circ} \mathrm{C} \text { during } \mathrm{HI} \text {, } \\
37 \text { vs. } 32^{\circ} \mathrm{C}, \\
\text { during } 5 \mathrm{~h} \text { of } \\
\text { recovery }\end{array}$ & $\begin{array}{l}\downarrow \text { infarct area in HI }+ \text { HT } \\
+ \text { Xe }(19 \%) \text { vs. HI }+ \text { HT } \\
(37 \%) \text { and NT }(54 \%), \\
p<0.05\end{array}$ & None & 1 week & $\sigma^{\prime} / 9$ \\
\hline 85 & $\begin{array}{l}\mathrm{P} 7 \\
\text { rats }\end{array}$ & $\begin{array}{l}\mathrm{HI}: 2 \mathrm{~h} \\
8 \% \mathrm{O}_{2}\end{array}$ & $\begin{array}{l}\text { I } \alpha I P(\text { inter- } \alpha \text { inhibitor } \\
\text { protein) (i.p.) } \\
\text { vehicle }+\mathrm{HI} \\
\text { human I } \alpha \mathrm{IP}+\mathrm{HI}\end{array}$ & $\begin{array}{l}\text { Pre-Tx: } \\
\text { immediately } \\
\text { before and } \\
24 \text { h after HI }\end{array}$ & Not specified & $\begin{array}{l}\text { Intermediate } \uparrow \text { cortical } \\
\text { and hippocampal tissue } \\
\text { vol. vs. vehicle }\end{array}$ & $\begin{array}{l}\text { Improved } \\
\text { spatial and nonspatial } \\
\text { learning vs. } \\
\text { vehicle }\end{array}$ & $\begin{array}{l}\text { Pathology: } \\
22 \text { weeks } \\
\text { ( } 157 \text { days) } \\
\text { behavior: } \\
11 \text { weeks } \\
\text { ( } 80 \text { days) }\end{array}$ & $\sigma^{x}$ \\
\hline 86 & $\begin{array}{l}\text { P7 } \\
\text { rats }\end{array}$ & $\begin{array}{l}\text { HI: } 55 \mathrm{~min} \\
8 \% \mathrm{O}_{2}\end{array}$ & $\begin{array}{l}\text { Estetrol (estrogenic steroid) } \\
\text { (i.p.) } \\
\text { vehicle + HI } \\
\text { estetrol } 1,5,10,50 \mathrm{mg} / \mathrm{kg} / \\
\text { day + HI }\end{array}$ & $\begin{array}{l}\text { Pre-Tx: } \\
-3 \text { days }\end{array}$ & $\begin{array}{l}\text { Ambient: } \\
37^{\circ} \mathrm{C} \text {, during } \mathrm{HI}\end{array}$ & $\begin{array}{l}\downarrow \text { infarct area in all } \\
\text { treatment groups vs. } \\
\text { vehicle, } p<0.05\end{array}$ & None & 1 week & $?$ \\
\hline 86 & $\begin{array}{l}\text { P7 } \\
\text { rats }\end{array}$ & $\begin{array}{l}\text { HI: } 55 \mathrm{~min} \\
8 \% \mathrm{O}_{2}\end{array}$ & $\begin{array}{l}\mathrm{HI}+\text { vehicle } \\
\mathrm{HI}+\text { estetrol 1, 5, 10, } 50 \\
\mathrm{mg} / \mathrm{kg}\end{array}$ & $\begin{array}{l}\text { Immediate } \\
\text { Tx: } 0 \mathrm{~h}\end{array}$ & $\begin{array}{l}\text { Ambient: } \\
37^{\circ} \mathrm{C} \text {, during } \mathrm{HI}\end{array}$ & $\begin{array}{l}\downarrow \text { infarct area in all } \\
\text { treatment groups vs. } \\
\text { vehicle }\end{array}$ & None & 1 week & $?$ \\
\hline 87 & $\begin{array}{l}\mathrm{P} 8 \\
\text { rats }\end{array}$ & $\begin{array}{l}\text { HI: } 45 \mathrm{~min} \\
7.7 \% \mathrm{O}_{2}\end{array}$ & $\begin{array}{l}\text { Lithium (i.p.) } \\
\mathrm{HI}+\text { vehicle } \\
\mathrm{HI}+\text { lithium }\end{array}$ & $\begin{array}{l}\text { Post-Tx: } \\
\text { 5-19 days }\end{array}$ & $\begin{array}{l}\text { Ambient: } \\
36^{\circ} \mathrm{C} \text {, during } \mathrm{HI}\end{array}$ & $\begin{array}{l}\downarrow \text { tissue vol loss } 169 \pm 26 \\
\text { vs. } 277 \pm 27 \mathrm{~mm}^{3} \text { and } \\
\text { pathological score, } \\
p<0.05 \text { vs. vehicle }\end{array}$ & $\begin{array}{l}\text { Improved motor- } \\
\text { and } \downarrow \text { anxiety- } \\
\text { related activity, } \\
p<0.05 \text { vs. vehicle }\end{array}$ & $\begin{array}{l}\text { Pathology: } \\
12 \text { weeks } \\
\text { behavior: } \\
6 \text { weeks }\end{array}$ & $\sigma^{x}$ \\
\hline 88 & $\begin{array}{l}\mathrm{P} 7 \\
\text { rats }\end{array}$ & $\begin{array}{l}\mathrm{HI}: 2 \mathrm{~h} \\
8 \% \mathrm{O}_{2}\end{array}$ & $\begin{array}{l}\text { Isoflurane or cyclosporine A } \\
\text { (mitochondrial permeability } \\
\text { transition pore inhibitor) } \\
\text { (i.c.v.) } \\
\text { HI + vehicle } \\
\text { HI + isoflurane } \\
\text { HI + cyclosporine A }\end{array}$ & $\begin{array}{l}\text { Immediate } \\
\text { Tx: } 0 \mathrm{~h}\end{array}$ & $\begin{array}{l}\text { Ambient: } \\
37^{\circ} \mathrm{C} \text {, during } \mathrm{HI}\end{array}$ & $\begin{array}{l}\uparrow \text { hippocampal and } \\
\text { thalamic neuronal density } \\
\text { in isoflurane and } \\
\text { cyclosporine A groups } \\
\text { vs. vehicle, } p<0.05\end{array}$ & & 1 week & $\sigma^{\prime} / 9$ \\
\hline 88 & $\begin{array}{l}\mathrm{P} 7 \\
\text { rats }\end{array}$ & $\begin{array}{l}\mathrm{HI}: 2 \mathrm{~h} \\
8 \% \mathrm{O}_{2}\end{array}$ & $\begin{array}{l}\mathrm{HI}+\text { vehicle } \\
\mathrm{HI}+\text { isoflurane } \\
\mathrm{HI}+\text { cyclosporine A }\end{array}$ & $\begin{array}{l}\text { Immediate } \\
\text { Tx: } 0 \mathrm{~h}\end{array}$ & $\begin{array}{l}\text { Ambient: } \\
37^{\circ} \mathrm{C} \text {, during } \mathrm{HI}\end{array}$ & $\begin{array}{l}\uparrow \text { hippocampal and } \\
\text { thalamic neuronal } \\
\text { density in isoflurane and } \\
\text { cyclosporine A groups } \\
\text { vs. vehicle, } p<0.05\end{array}$ & $\begin{array}{l}\text { Similar improvement in } \\
\text { spatial learning and } \\
\text { memory in isoflurane and } \\
\text { cyclosporine A groups vs. } \\
\text { vehicle }\end{array}$ & 5 weeks & $\sigma^{\top} / 9$ \\
\hline 89 & $\begin{array}{l}\text { P3-5 } \\
\text { piglets }\end{array}$ & $\begin{array}{l}45 \text { min } \\
\mathrm{FiO}_{2} 10 \% \\
\text { then } \\
\text { asphyxia } \\
7 \text { min }\end{array}$ & $\begin{array}{l}\text { HT and HETE-0016 } \\
\text { (20-hydroxyeicosatetraeonic } \\
\text { acid inhibitor) (i.v.) } \\
\text { Hypox }+ \text { NT } \\
\text { Hypox }+ \text { HT } \\
\text { Hypox }+ \text { HETE- } 0016+\text { HT }\end{array}$ & $\begin{array}{l}\text { Post-Tx: } \\
\text { HETE-0016 } \\
\text { at } 5 \mathrm{~min} \\
\mathrm{HT} \text { at } 3 \mathrm{~h}\end{array}$ & $\begin{array}{l}\text { Core: } \\
38.5-39.5 \text { vs. } \\
34.0^{\circ} \mathrm{C} \\
\text { continuous } \\
\text { monitoring }\end{array}$ & $\begin{array}{l}\uparrow \text { neuronal survival in } \\
\text { cortex }(\sim 22 \%) \text {, putamen } \\
(\sim 14 \%) \text {, and thalamus } \\
(\sim 28 \%) \text { with combination } \\
\text { therapy, } p<0.05 \text { vs. HT }\end{array}$ & $\begin{array}{l}\text { No effect on time to feed } \\
\text { after extubation; no } \\
\text { significant effect on } n \text { of } \\
\text { clinical seizures, } p<0.1\end{array}$ & $\begin{array}{l}1 \text { week } \\
\text { (10 days) }\end{array}$ & $\sigma^{x}$ \\
\hline
\end{tabular}


Table 1 (continued)

\begin{tabular}{|c|c|c|c|c|c|c|c|c|c|}
\hline $\begin{array}{l}\text { Refer- } \\
\text { ence }\end{array}$ & Species & Insult & Treatment (route) & Timing & Temperature, ${ }^{\circ} \mathrm{C}$ & Pathology & $\begin{array}{l}\text { Functional } \\
\text { outcome }\end{array}$ & Survival & Sex \\
\hline \multicolumn{10}{|c|}{ No improvement in neurodevelopmental outcomes } \\
\hline 90 & $\begin{array}{l}\text { P7 } \\
\text { rats }\end{array}$ & $\begin{array}{l}\mathrm{HI}: 150 \mathrm{~min} \\
8 \% \mathrm{O}_{2}\end{array}$ & $\begin{array}{l}\text { HT and Xe } \\
\text { NT } \\
\mathrm{HT} \\
\mathrm{HT}+\mathrm{Xe}\end{array}$ & $\begin{array}{l}\text { Immediate } \\
\text { Tx: } 0 \mathrm{~h}\end{array}$ & $\begin{array}{l}\text { Core: } \\
37 \text { vs. } 32^{\circ} \mathrm{C} \text {, } \\
\text { during } 5 \mathrm{~h} \text { of } \\
\text { recovery }\end{array}$ & $\begin{array}{l}\text { No effect of HT or HT + } \\
\text { Xe on infarct vol and } \\
\text { neuronal loss vs. NT }\end{array}$ & & 1 week & $\sigma^{\pi} / 9$ \\
\hline 55 & $\begin{array}{l}\mathrm{P} 7 \\
\text { rats }\end{array}$ & $\begin{array}{l}\mathrm{HI}: 2 \mathrm{~h} \\
15 \mathrm{~min} \\
8 \% \mathrm{O}_{2}\end{array}$ & $\begin{array}{l}\text { Resveratrol (i.p.) } \\
\mathrm{HI}+\text { vehicle } \\
\mathrm{HI}+\text { resveratrol }\end{array}$ & $\begin{array}{l}\text { Immediate } \\
\text { Tx: } 0 \mathrm{~h}\end{array}$ & $\begin{array}{l}\text { Ambient: } \\
36^{\circ} \mathrm{C} \text {, during } \mathrm{HI}\end{array}$ & $\begin{array}{l}\text { No effect on infarct vol, } \\
\text { neuronal loss or } \\
\text { myelination vs. vehicle }\end{array}$ & None & 1 week & $\sigma^{2} / 9$ \\
\hline 91 & $\begin{array}{l}\text { P9 } \\
\text { mice }\end{array}$ & $\begin{array}{l}\text { HI: } 50 \mathrm{~min} \\
10 \% \mathrm{O}_{2}\end{array}$ & $\begin{array}{l}\text { Osteopontin (i.n./i.p.) } \\
\mathrm{HI}+\text { vehicle } \\
\mathrm{HI}+\text { osteopontin }\end{array}$ & $\begin{array}{l}\text { Immediate } \\
\text { and post-Tx: } \\
0 \text {, and } 3 \mathrm{~h} \text { and } \\
1-3 \text { days i.n. } \\
\text { or } 0 \text { h and every } \\
2 \text { days on days } \\
1-15 \text { i.p. or } \\
0 \text { h and every } \\
2 \text { days on days } \\
5-15 \text { i.p. }\end{array}$ & $\begin{array}{l}\text { Ambient: } \\
37^{\circ} \mathrm{C} \text {, during } \mathrm{HI}\end{array}$ & $\begin{array}{l}\text { No effect on white (MBP) } \\
\text { or gray (MAP2) matter } \\
\text { area in any treatment } \\
\text { group vs. vehicle; } \\
\text { no sex-specific effect }\end{array}$ & $\begin{array}{l}\text { No effect on } \\
\text { sensorimotor } \\
\text { performance }\end{array}$ & $\begin{array}{l}\text { Pathology: } \\
9 \text { weeks } \\
\text { ( } 65 \text { days) } \\
\text { behavior: } \\
8 \text { weeks } \\
\text { ( } 60 \text { days) }\end{array}$ & $\sigma^{2} / 9$ \\
\hline 23 & $\begin{array}{l}\text { Fetal } \\
\text { sheep } \\
(0.85 \\
\text { GA })\end{array}$ & $\begin{array}{l}\text { Bilateral } \\
\text { carotid artery } \\
\text { occlusion } \\
30 \text { min }\end{array}$ & $\begin{array}{l}H T \\
\text { ischemia + NT } \\
\text { ischemia + HT } 3 \text { days } \\
\text { ischemia + HT } 5 \text { days }\end{array}$ & $\begin{array}{l}\text { Post-Tx: } \\
3 \mathrm{~h}\end{array}$ & $\begin{array}{l}\text { Brain: } \\
39.5 \text { vs. } 31.3- \\
31.8^{\circ} \mathrm{C} \\
\text { continuous } \\
\text { monitoring }\end{array}$ & $\begin{array}{l}\downarrow \text { neuronal survival in } \\
\text { cortex and dentate gyrus } \\
\text { in 5- vs. 3-h HT group, } \\
p<0.05\end{array}$ & $\begin{array}{l}\text { Improved EEG recovery } \\
\text { and } \downarrow \text { brain edema in } \\
3 \text { - and } 5 \text {-day groups, } \\
p<0.05 \text { vs. NT }\end{array}$ & 1 week & $\sigma^{2} / 9$ \\
\hline 22 & $\begin{array}{l}\text { Fetal } \\
\text { sheep } \\
(0.85 \\
\text { GA })\end{array}$ & $\begin{array}{l}\text { Bilateral } \\
\text { carotid artery } \\
\text { occlusion } \\
30 \text { min }\end{array}$ & $\begin{array}{l}\text { HT and Cx43 (connexin } \\
\text { hemichannel blockade) } \\
\text { (i.c.v.) } \\
\text { ischemia }+ \text { NT } \\
\text { ischemia }+ \text { HT } \\
\text { ischemia }+\mathrm{HT}+\mathrm{Cx} 43\end{array}$ & $\begin{array}{l}\text { Post-Tx: } \\
3 \mathrm{~h}\end{array}$ & $\begin{array}{l}\text { Brain: } \\
39.5 \text { vs. } 31.3- \\
32.1^{\circ} \mathrm{C} \\
\text { continuous } \\
\text { monitoring }\end{array}$ & $\begin{array}{l}\text { No additive protection } \\
\text { with } \mathrm{Cx} 43 \text { block }+\mathrm{HT} \\
\text { vs. HT }\end{array}$ & $\begin{array}{l}\text { No additive effect with } \\
\text { Cx } 43 \text { block + HT on EEG } \\
\text { recovery or brain edema } \\
\text { vs. HT }\end{array}$ & 1 week & $\sigma^{2} / 9$ \\
\hline 92 & $\begin{array}{l}\text { P1 } \\
\text { piglets }\end{array}$ & $\begin{array}{l}45 \mathrm{~min} \\
\mathrm{FiO}_{2} 6 \%\end{array}$ & $\begin{array}{l}\text { HT } \\
\text { (selective head cooling) } \\
\text { Hypox + NT } \\
\text { Hypox + HT }\end{array}$ & $\begin{array}{l}\text { Immediate } \\
\text { Tx: } 0 \mathrm{~h}\end{array}$ & $\begin{array}{l}\text { Brain: } \\
38.5 \text { vs. } 31^{\circ} \mathrm{C} \text {, } \\
\text { continuously for } \\
24 \text { h then daily }\end{array}$ & $\begin{array}{l}\text { No improvement in } \\
\text { neuropathology scores in } \\
\text { cortical and subcortical } \\
\text { gray or white matter }\end{array}$ & $\begin{array}{l}\text { No difference in } n \text { of } \\
\text { electrographic or clinical } \\
\text { seizures between groups }\end{array}$ & 3 days & $\sigma^{2} / 9$ \\
\hline 63 & $\begin{array}{l}\mathrm{P} 7 \\
\text { rats }\end{array}$ & $\begin{array}{l}\mathrm{HI}: 150 \mathrm{~min} \\
8 \% \mathrm{O}_{2}\end{array}$ & $\begin{array}{l}\text { Vitexin }(5,7, \\
\text { 4-trihydroxyflavone-8- } \\
\text { glucoside) (i.p.) } \\
\text { HI + vehicle } \\
\text { HI + vitexin }\end{array}$ & $\begin{array}{l}\text { Post-Tx: } \\
3 \mathrm{~h}\end{array}$ & $\begin{array}{l}\text { Ambient: } \\
37^{\circ} \mathrm{C} \text {, during } \mathrm{HI}\end{array}$ & $\begin{array}{l}\text { No effect on infarct vol } \\
\text { vs. vehicle }\end{array}$ & None & 2 days & $\sigma^{2} / 9$ \\
\hline$\overline{93}$ & $\begin{array}{l}\mathrm{P} 7 \\
\text { rats }\end{array}$ & $\begin{array}{l}\text { LPS + HI: } \\
50 \mathrm{~min} \\
8 \% \mathrm{O}_{2}\end{array}$ & $\begin{array}{l}H T \\
\mathrm{LPS}+\mathrm{HI}+\mathrm{NT} \\
\mathrm{LPS}+\mathrm{HI}+5 \mathrm{~h} \mathrm{HT}\end{array}$ & $\begin{array}{l}\text { Immediate } \\
\text { Tx: } 0 \mathrm{~h}\end{array}$ & $\begin{array}{l}\text { Core: } \\
37 \text { vs. } 32^{\circ} \mathrm{C} \text {, } \\
\text { during } 5 \mathrm{~h} \text { of } \\
\text { recovery }\end{array}$ & $\begin{array}{l}\text { No effect on hippocampal } \\
\text { neuronal loss vs. NT }\end{array}$ & None & 1 week & $\sigma^{2} / 9$ \\
\hline 94 & $\begin{array}{l}\text { P3-5 } \\
\text { piglets }\end{array}$ & $\begin{array}{l}\mathrm{HI} 45 \mathrm{~min} \\
10 \% \mathrm{O}_{2} \\
\text { then } \\
\text { asphyxia } \\
7 \text { min } \\
\end{array}$ & $\begin{array}{l}H T \\
\mathrm{HI}+\mathrm{NT} \\
\mathrm{HI}+\mathrm{HT} \\
\mathrm{HI}+\mathrm{HT} \text { slow rewarming } \\
\text { HI + HT fast rewarming }\end{array}$ & $\begin{array}{l}\text { Post-Tx: } \\
2 \mathrm{~h}\end{array}$ & $\begin{array}{l}\text { Core: } \\
38.5-39.5 \text { vs. } \\
34.0^{\circ} \mathrm{C} \text {, } \\
\text { continuous } \\
\text { monitoring }\end{array}$ & $\begin{array}{l}\uparrow \text { cortical neuronal } \\
\text { apoptosis in both } \\
\text { rewarming groups vs. HT } \\
\text { and NT groups, } p<0.05\end{array}$ & None & 1 day & $\sigma^{x}$ \\
\hline 95 & $\begin{array}{l}\text { P7 } \\
\text { rats }\end{array}$ & $\begin{array}{l}\mathrm{LPS}+\mathrm{HI} \\
50 \mathrm{~min} \\
8 \% \mathrm{O}_{2}\end{array}$ & $\begin{array}{l}H T \\
\mathrm{LPS}+\mathrm{HI}+5 \mathrm{~h} \mathrm{NT} \\
\mathrm{LPS}+\mathrm{HI}+5 \mathrm{~h} \mathrm{HT}\end{array}$ & $\begin{array}{l}\text { Immediate } \\
\text { Tx: } 0 \mathrm{~h}\end{array}$ & $\begin{array}{l}\text { Core: } \\
36^{\circ} \mathrm{C} \text { during HI } \\
37 \text { vs. } 32^{\circ} \mathrm{C} \text { during } \\
5 \text { h of recovery }\end{array}$ & $\begin{array}{l}\text { No effect on \% brain area } \\
\text { loss, HT vs. NT }\end{array}$ & None & 1 week & $\sigma^{2} / 9$ \\
\hline 84 & $\begin{array}{l}\text { P7 } \\
\text { rats }\end{array}$ & $\begin{array}{l}\text { HI: } 90 \mathrm{~min} \\
8 \% \mathrm{O}_{2}\end{array}$ & $\begin{array}{l}\mathrm{HI}+\mathrm{NT} \\
\mathrm{HI}+5 \mathrm{~h} \text { HT } 35^{\circ} \mathrm{C} \\
\mathrm{HI}+5 \mathrm{~h} \text { HT } 35^{\circ} \mathrm{C}+ \\
\mathrm{Xe} 20 \%\end{array}$ & $\begin{array}{l}\text { Post-Tx: } \\
4 \mathrm{~h}\end{array}$ & $\begin{array}{l}\text { Core: } 36^{\circ} \mathrm{C} \text {, during } \\
\text { HI } 37 \text { vs. } 32^{\circ} \mathrm{C} \text {, } \\
\text { during } 5 \mathrm{~h} \text { of } \\
\text { recovery }\end{array}$ & $\begin{array}{l}\text { No effect of delayed } \\
\text { combination or HT alone } \\
\text { on infarct area vs. NT }\end{array}$ & None & 1 week & $\sigma^{2} / 9$ \\
\hline
\end{tabular}

aEEG, amplitude-integrated electroencephalogram; DHA, docosahexaenoic acid; GA, gestational age; G-CSF, granulocyte colony stimulating factor; HT, hypothermia; HI, hypoxia-ischemia; Hypox: hypoxia; HPC, hypoxic preconditioning; iNOS, inducible nitric oxide synthase; LPS, lipopolysaccharide; MBP, myelin basic protein; NAA, N-acetyl aspartate; NAC, $\mathrm{N}$-acetyl-L-cysteine; NeuN, neuronal nuclear protein; NT, normothermia; nNOS: neuronal nitric oxide synthase; P, postnatal day; P5-TAT, cycline-dependent kinase 5 inhibitor; Tx, treatment; UCO, umbilical cord occlusion; vol, volume; WT, wild type; Xe, xenon.

\section{Studies of Perinatal Neuroprotection}

We identified and screened a total of 171 papers. One hundred and ten were excluded due to inappropriate developmental age, ex vivo assessment, or lack of assessment of neurological outcomes. Thus 61 individual pa- pers were included in this analysis (Fig. 1). Studies were further subdivided by treatment regime (i.e., studies that investigated more than 1 timing for treatment relative to the insult) or where pathological and functional outcomes were assessed at different survival times. After
Temperature Control and Neuroprotection
Dev Neurosci 2017;39:7-22

DOI: $10.1159 / 000452859$ 


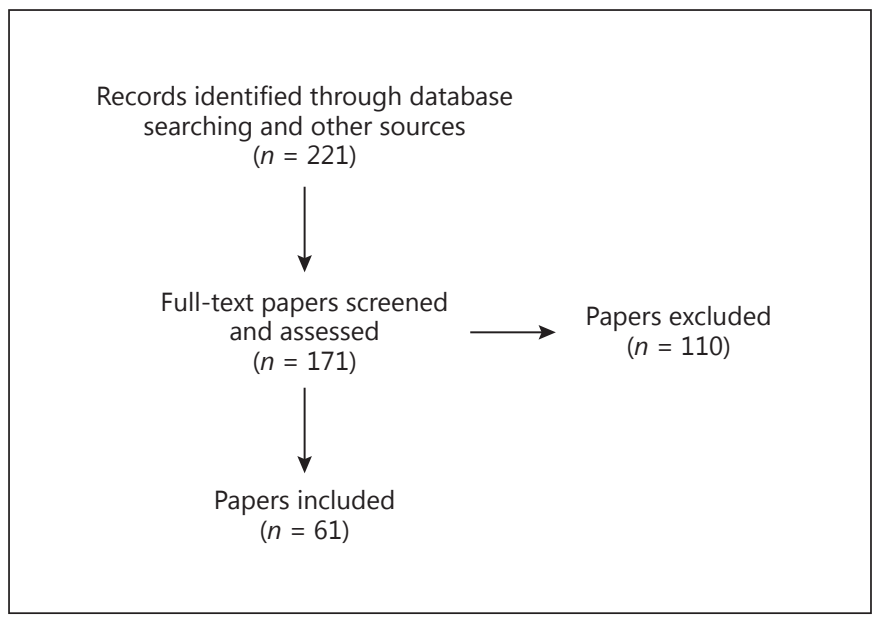

Fig. 1. Flow chart illustrating the number of papers identified through database searching and other relevant sources, the number of full-text articles screened, assessed, and excluded, and the final number of original papers surveyed.

subdividing the studies that used more than 1 survival time for pathological and functional assessments or assessed outcomes after more than 1 treatment time, we identified 75 individual studies. For the purpose of reporting on the treatment regime, timing, outcomes, and survival, we summarized the data based on the individual studies (total $n=75$ ). As each of the subdivided studies used the same developmental age at the time of the insult and protocols for temperature control and documentation of sex, the developmental age, type and duration of temperature monitoring, and reporting of sex are summarized based on the number of original papers (total $n=61)$.

The majority of papers 52/61 (85\%) studied neonatal rats or mice; 35 out of these $52(67 \%)$ papers studied neonatal rodents at $\mathrm{P} 7$, the remainder studied rodents between P8 and P11 (17/52; 32\%). These ages are broadly comparable to the late preterm or term human infant at 32-40 weeks [17-19]. All studies used the Rice-Vannucci model of unilateral carotid artery ligation followed by a period of moderate hypoxia [18]. A range of pathological assessments of injury was reported, such as measurements of total and/or regional infarct area or volume or semiquantitative assessments to assign a neuronal injury score. Functional outcomes were assessed using a wide variety of behavioral tests examining memory, sensorimotor function, coordination, or neophobia.

Nine out of 61 papers (15\%) studied large animals. Histopathological assessments were mainly performed by quantifying the total number of viable or dying cells within regions susceptible to injury. In term-equivalent fetal sheep, injury was induced using bilateral carotid artery occlusion or repeated umbilical cord occlusion. In the piglet, injury was induced using prolonged isocapnic hypoxia with or without a short (7-min) period of severe asphyxia or bilateral carotid artery occlusion. In both paradigms, injury has been reported in "watershed" zones (areas of white and gray matter that lie within the borders between major arteries where perfusion pressure is least), such as the parasagittal cortex, the dorsal horn of the hippocampus, thalamus, dentate gyrus, cerebellar neocortex, striatum, and parasagittal and periventricular white matter. Functional outcomes were assessed using electroencephalography (EEG) to evaluate recovery of activity and/or electrographic or clinical seizure assessment. In neonatal piglets, clinical seizures were also assessed. In fetal sheep, cortical impedance was used to examine the degree of cytotoxic edema after the insult.

\section{Results}

\section{Temperature Monitoring}

In 7 of 61 papers, temperature monitoring was not reported in the study design or outcomes. Of these, 6 were in rodents and 1 was in fetal sheep. All of these papers reported neuroprotection (Fig. 1a). Environmental temperature only was reported in 32 papers (all in rodents); 31 out of these 32 papers reported neuroprotection, including 2 papers that tested hypothermia, and 1 paper reported no improvement in histological or functional outcomes. Twenty-eight of these 32 papers reported environmental temperature during $\mathrm{HI}$ only (Fig. 2a, 3a).

Core temperature was monitored in 19/61 papers (31\%; 14 papers used rodents and 5 papers used piglets; Fig. 2). In rodents, $1 / 14$ papers monitored temperature intermittently for 7 days (Fig. 3), 2 papers monitored core temperature during $\mathrm{HI}$, and 2 papers monitored core temperature during $\mathrm{HI}$ for 30 min or $2 \mathrm{~h}$ afterwards; all of these papers reported improved outcomes with treatment. Nine papers in rodents monitored core temperature for 3-10 h after HI (Fig. 3c). Of these, 6/9 papers reported neuroprotection at least in some treatment groups.

In all papers using piglets, core temperature was monitored continuously (Fig. 3b). All of these papers focused on hypothermia strategies or adjuvant therapy with hy-
14

Dev Neurosci 2017;39:7-22

DOI: $10.1159 / 000452859$
Galinsky/Dean/Lear/Davidson/Dhillon/ Wassink/Bennet/Gunn 
Fig. 2. Number of papers that did not monitor temperature (none) and papers that reported ambient (environmental), core, and brain temperature $(\mathrm{T})$ in rodents $(\mathbf{a})$ and large animals (b) from 61 papers investigating neuroprotection for term/near-term HIE between January 1, 2014, and June 30, 2016. Open bars show the number of studies that reported no protection. Black bars show the number of studies that reported neuroprotection.
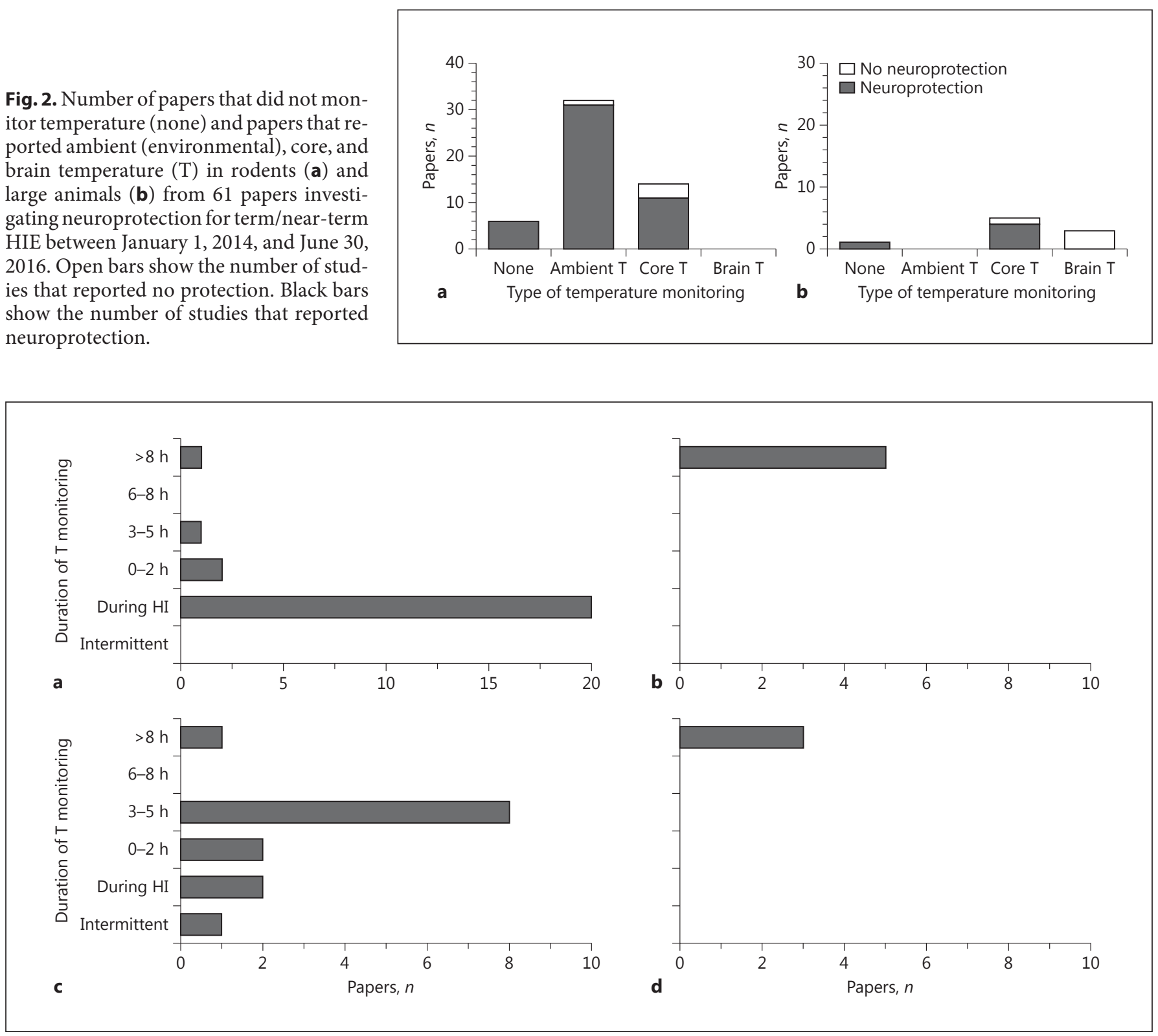

Fig. 3. Number of papers in rodents $(n=39 ; \mathbf{a}, \mathbf{c})$ and large animals $(n=7 ; \mathbf{b}, \mathbf{d})$ that reported the duration of temperature (T) monitoring: ambient/environmental (a) and core temperature (c) monitoring in rodents, and core (b) and brain temperature (d) monitoring in large animals. Total $n=46$.

pothermia, and all reported at least partial protection. Brain temperature was monitored in $3 / 61$ papers $(5 \% ; 1$ in piglets and 2 in fetal sheep; Fig. 3d). In the piglet study, brain temperature was monitored for $24 \mathrm{~h}$ and then once daily for 2 days. In 2 papers using fetal sheep, extradural brain temperature was monitored continuously throughout the experiment.

Temperature Control and

Neuroprotection

\section{Possibility that Neuroprotectants Affected}

\section{Thermoregulation}

We identified a total of 45 neuroprotectants/protective strategies from the papers reviewed in this study. Twenty treatments were classified as likely to affect thermoregulation, and 5 were considered unlikely to affect temperature control. The effect on thermoregulation was unknown in 20 cases (Fig. 4a).

Dev Neurosci 2017;39:7-22 DOI: $10.1159 / 000452859$ 

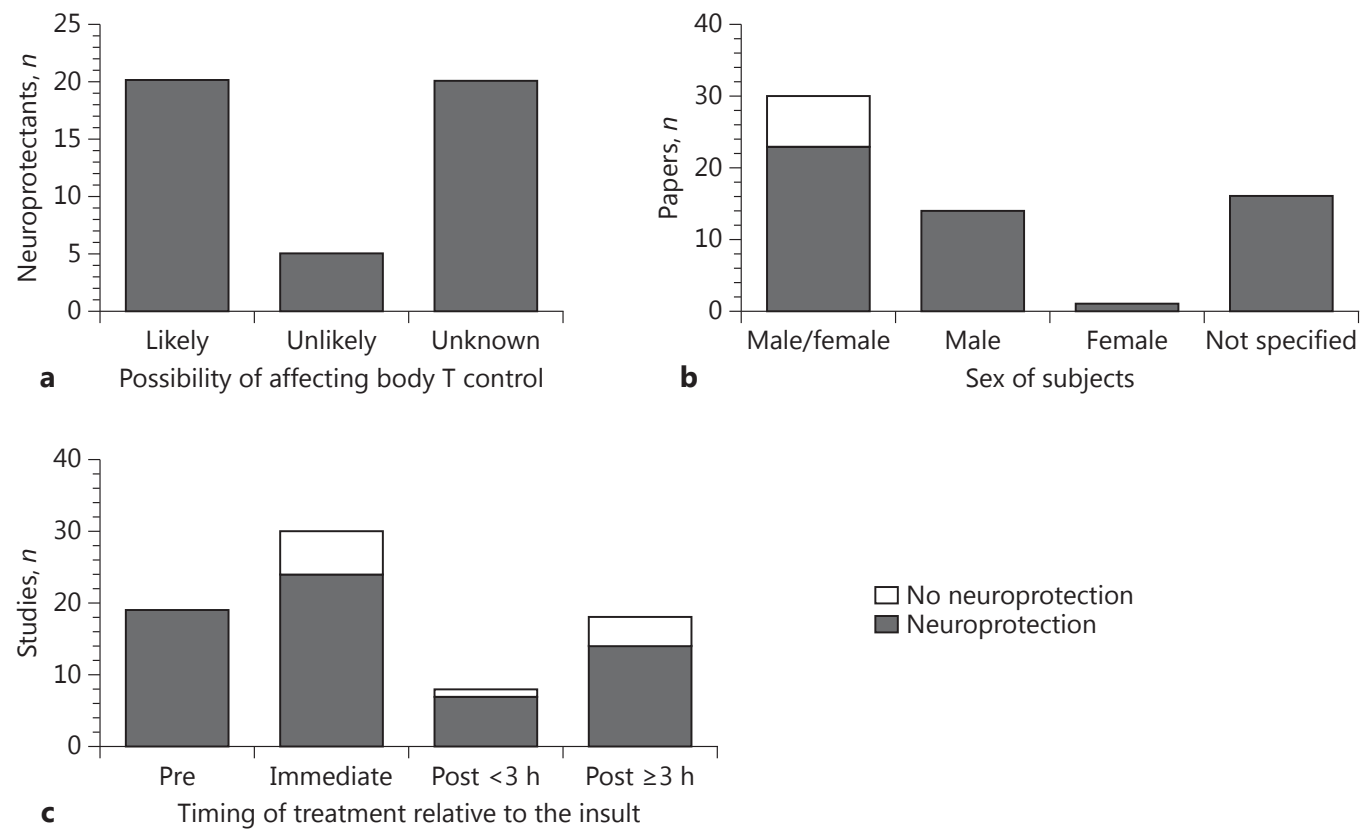

$\square$ No neuroprotection $\square$ Neuroprotection

Fig. 4. a Probability of neuroprotectants affecting thermoregulation; 36 neuroprotectants were identified and assessed. Likely was defined as a drug previously shown to affect temperature (T) or belonging to a family of drugs reported to affect temperature. Unlikely refers to a drug previously shown to have no effect on temperature. Unknown indicates no studies have investigated whether the drug/neuroprotective strategy affects temperature. b Number of papers that reported no neuroprotection (white bars) or neuroprotection (black bars) in either both sexes, males, or females, or did not report the sex of the subjects studied. c Number of studies that reported no neuroprotection (white bars) or neuroprotection (black bars) after treating before, immediately, within less than $3 \mathrm{~h}$, or greater than or equal to $3 \mathrm{~h}$ after the insult.
Fig. 5. Number of studies that reported no protection (white bars) or neuroprotection (black bars) after using short ( $\leq 1-10$ days), medium (20-77 days), or long (80-154 days) survival times for histological (a) and behavioral assessment (b).

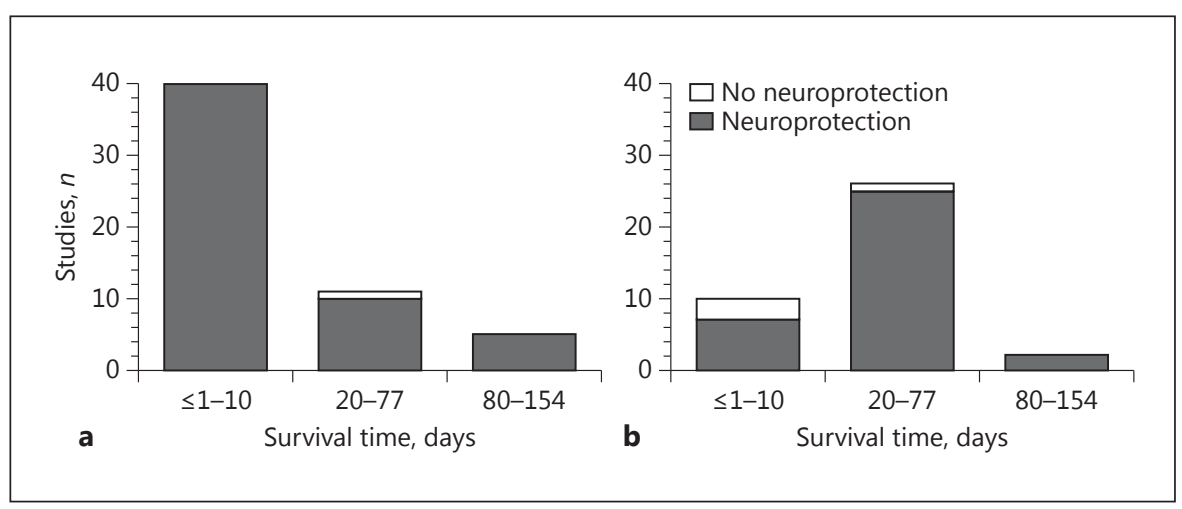

\section{Route of Drug Delivery}

The majority of the papers surveyed $(52 / 61 ; 85 \%)$ assessed the effectiveness of therapeutic candidates without hypothermia. Nine out of 61 papers (15\%) investigated hypothermia with adjuvant therapies. Twelve out of 61 papers $(20 \%)$ investigated hypothermia alone. The treat- ment regimens for candidate drugs were highly variable. Pharmacological interventions were administered in either single or multiple doses. Most of the rodent studies administered candidate treatments subcutaneously or intra-peritoneally $(n=32)$. One rodent study used a combination of intraperitoneal and intra-nasal drug delivery, 
and 12 studies delivered candidate drugs in gaseous form during or after HI. Eight studies (7 in rodents and 1 in fetal sheep) used intracerebroventricular drug delivery, 2 studies (both in rodents) delivered the drug as a dietary supplement, 1 study in fetal sheep administered the candidate drug via the maternal circulation, and 1 study in neonatal piglets used intravenous infusion. Two rodent studies used a constitutive gene knockout.

Sex

Thirty of 61 papers (49\%) examined the effect of treatment on both sexes (Fig. 4b). Twenty-three studies showed improved neural outcomes, and 7 studies showed no improvement. In a further 14 papers, therapeutic candidates were examined in males, and 1 paper reported outcomes in females only. All reported improved neural outcomes. Finally, in 16 papers, the sex of the subjects was not reported; all of these studies reported neuroprotection.

\section{Timing of Treatment and Neurodevelopmental Outcomes}

In total, 64/75 studies demonstrated neuroprotection for HIE. In 19 studies, treatment began before the start of HI (Fig. 4c), including two studies of constitutive gene knockouts (i.e. before HI). In 31 studies, treatment was delivered during or immediately after $\mathrm{HI}$ or within $2 \mathrm{~h}$. In just 14 studies, treatment was started $3 \mathrm{~h}$ or later after HI. The survival time before histological assessment in 44 studies reporting neuroprotection was less than or equal to 1-10 days (Fig. 5a). In 10 studies, histological outcomes were reported between 20 and 77 days. In 5 studies, outcomes were reported between 80 and 154 days. Five studies did not report histological outcomes.

Thirty-four studies (32 in rodents and 2 in piglets) reported improved functional outcomes. Of these, 7 assessed functional outcomes after less than or equal to 1-10 days, 25 after 20-77 days, and 2 after 80-154 days (Fig. 5b). Interestingly, in 5 studies, histological and functional outcomes were discordant, such that 3 studies reported improved histology with no improvement in function, and 2 studies found improved functional outcomes without improved histology.

Eleven out of 75 perinatal studies reported no neuroprotection with treatment. Seven studies began treatment immediately after $\mathrm{HI}$ (or within the first $2 \mathrm{~h}$ ), and 4 studies delayed treatment for up to 3-4 h after HI (Fig. 4c). In 10 of the 11 studies, histological analysis was performed after relatively short survival times of less than or equal to 1-10 days. One study in rodents examined histological

Temperature Control and

Neuroprotection outcomes after approximately 9 weeks (65 days; Fig. 5a). Four studies assessed functional outcomes. Three studies ( 1 in piglets and 2 in fetal sheep) assessed functional outcomes based on EEG or clinical seizures after less than or equal to 1-10 days, and 1 study in rodents examined behavioral outcomes after 60 days (Fig. 5b).

\section{Discussion}

This systematic review shows that the majority of recent studies investigating perinatal neuroprotection for HIE between 2014 and 2016 were performed in rodents, of which only a minority rigorously monitored and controlled body and brain temperatures. Thus, many recent studies cannot exclude the possibility that their findings could have been confounded by unappreciated changes in brain temperature due to drug-induced cooling or environmental conditions.

Carefully conducted studies in neonatal rodents offer many advantages for studies of neuroprotection [17]. They are easy to use and cost effective, which in turn facilitates data collection from large cohorts at multiple time points. Further, rodent studies enable wide application of histological techniques, molecular biology probes, and behavioral assessments, allowing investigators to provide detailed mechanistic and functional insight. However, as highlighted in this systematic review, considerable care is required to be certain that iatrogenic changes in brain temperature do not occur. Large animal studies are expensive, require more laboratory infrastructure relative to studies in smaller animals, and offer limited opportunities for assessing neurodevelopmental outcomes other than neurophysiological monitoring. Nevertheless, they are easier to monitor and have intrinsically greater thermal stability.

The great majority of recent papers used neonatal mice or rats (53/61). Only 14 papers studying rodents measured body temperature at all, and just 9 continuously monitored body temperature for short periods of 3-10 h after HI. No studies reported on temperatures after this early phase of recovery. Given the near-poikilothermic nature of P7 neonatal rats and mice, environmental temperature is often used as a surrogate for body temperatures. However, although most of the studies measured environmental temperature during the insult, few monitored or reported temperature after HI. This is an important consideration since even normal, healthy neonatal rodents often show lower body temperatures in the typical laboratory nesting conditions between P7

Dev Neurosci 2017;39:7-22

DOI: $10.1159 / 000452859$ 
and P14 [20]. Core temperature in neonatal rodents will be affected by the amount and composition of nesting material, position in the nest, huddling, distance from the dam, and the time since the last period of suckling. Conversely, the majority of large animal studies continuously monitored and controlled brain and or core temperature throughout the study period. One study did not report temperature in near-term fetal sheep; however, a major advantage of testing potential neuroprotectants in fetal sheep is that body temperature is regulated by the pregnant ewe and, therefore, unless the ewe is febrile, fetal temperatures are highly stable [21-24].

In adult rodents, the use of anesthetics such as halothane, sodium pentobarbital, and isoflurane around the time of the insult have all been shown to induce hypothermia, as previously reviewed [11]. Furthermore, many rodent studies have reported that the beneficial effects of potential neuroprotectants were confounded by hypothermia during the first $6-8 \mathrm{~h}$ after the insult $[11,25]$ and that maintenance of normothermia abolishes neuroprotection [25]. In this study, the majority $(40 / 45 ; 89 \%)$ of the pharmacological interventions being tested over the last 2 years belonged to classes of drugs that are either likely to affect body temperature or whose effect on thermoregulation is unknown. Collectively, these data strongly suggest that significant age-related and drug-induced fluctuations in body temperature, which might affect the severity of neural injury and, therefore, could confound studies of perinatal neuroprotection, may have been missed in the majority of recent studies.

The relative maturity of the species being studied is important for understanding the potential clinical application of the studies. The brain maturation of near-term fetal sheep (0.8-0.85 of gestation) and neonatal piglets is considered to be consistent with term human infants [26, 27]. However, at $P 7$, the rat is closest to the 32- to 36-weekold human infant based on the immaturity of the germinal zone, the limited extent of cortical layering, and reduced density of synapses in the striate cortex $[18,28]$. The majority of recent reports of neuroprotection in rodents (64\%) studied P7 rats. As recently reviewed, neonatal rats are closest to term equivalent at P10-P14 [17]. The responses to $\mathrm{HI}$ at $\mathrm{P} 10$ have been carefully characterized [29]. Even at P10-P14, healthy neonatal rodents often show significant spontaneous central hypothermia in the typical laboratory nesting environment [20], and therefore they still require continuous core temperature monitoring and control.
About half $(31 / 61 ; 51 \%)$ of the papers surveyed in this review reported outcomes either for only one sex or did not specify the sex of the subjects used. Preclinical studies have reported sexual dimorphisms in the severity and evolution of injury after $\mathrm{HI}$ and in responses to treatment, as previously reviewed [30]. For example, in P7 rats, males exposed to $\mathrm{HI}$ had greater deficits in some but not all behavioral tasks compared to females [30]. Similarly, there is some evidence of more variable responses to cooling in female mice [31]. It remains unclear whether there are similar differences in humans [32]. However, these data demonstrate the importance of carefully testing therapeutic agents in both sexes, as recommended by the National Institutes of Health [33].

A key clinical-translation consideration for testing potential neuroprotectants is when to treat. The majority of studies surveyed $(57 / 75 ; 76 \%)$ started intervention either before or immediately after the insult. Scientifically, since the mechanisms of intra-insult treatment are different from those after the insult cell death, efficacy during pretreatment does not necessarily suggest a benefit after the insult. Clinically, because most HI occurs around birth, it is very difficult to identify fetuses who are likely to develop postnatal HIE in advance as the positive predictive value of heart rate monitoring in labor is exceedingly low [34]. It remains a formidable challenge to start any intervention even within the first $6 \mathrm{~h}$ after birth [35]. Thus, in most cases, it is very difficult to translate preclinical neuroprotective agents until the window of opportunity after $\mathrm{HI}$ is known. Collectively, these data support evaluation of delayed treatment after the insult as part of routine preclinical evaluation.

It is still important to test the effectiveness of potential neuroprotectants during normothermia to help understand the mechanism/s by which they induce neuroprotection and to evaluate their potential for use in patients who may not be suitable candidates for therapeutic hypothermia. Nevertheless, given that hypothermia is now standard clinical practice $[35,36]$, ultimately it is essential to also test the effectiveness of potential drug therapies as adjuvants to therapeutic hypothermia in order to improve current treatment protocols. In this analysis, just 9 out of the 61 papers surveyed (15\%) assessed the effectiveness of hypothermia with adjuvant therapies.

Finally, the majority of studies $(54 / 75 ; 72 \%)$ examined relatively short survival times, from $\leq 1$ to 10 days. Less than half of the studies reported functional outcomes after treatment. Shorter survival times provide crucial information about the early outcome of injury and any intervention. Equally, particularly after moderate insults,
18

Dev Neurosci 2017;39:7-22

DOI: $10.1159 / 000452859$
Galinsky/Dean/Lear/Davidson/Dhillon/ Wassink/Bennet/Gunn 
injury can continue to evolve over many weeks [37], and behavioral and histological outcomes are sometimes discordant, as noted in this analysis.

This paper examined a sample of preclinical publications investigating neuroprotection between 2014 and 2016. It is not possible to know whether the methodological issues identified in our analysis affected the outcome of the studies. Nevertheless, the findings reported in this study are highly consistent with previous reports of the "adult" literature [38]. If this critical information is not presented in the publications, it is not possible to assess the significance of past and future studies in a meaningful way. Finally, we did not examine papers assessing mechanisms of HIE. It is very likely that these variables are just as important to understand the mechanisms of HIE and, therefore, it would be well worth analyzing them in the future.

\section{Conclusion}

The majority of recent studies of perinatal neuroprotection reported limited, or even no, assessment or maintenance of brain or body temperature. In addition, many did not address pragmatic treatment regimes, report or test the sex of the subjects, or assess long-term functional and histological outcomes. It is critical that all future preclinical studies of the mechanisms and treatment of perinatal HIE should carefully control brain and core temperatures during and after $\mathrm{HI}$.

\section{Contributor's Statement}

Robert Galinsky, Justin M. Dean, and Alistair J. Gunn conceptualized and designed the study. Robert Galinsky performed the initial search and analysis, and drafted the paper. Christopher A. Lear, Joanne O. Davidson, Simerdeep Dhillon, Guido Wassink, Laura Bennet, and Alistair J. Gunn provided in-depth review of the studies identified in the search and revised the paper. All authors critically reviewed the paper and approved the final version as submitted and agree to be accountable for all aspects of the work.

\section{Funding Sources}

This study was supported by the Health Research Council of New Zealand, Auckland Medical Research Foundation, and National Health and Medical Research Council (RG: 1090890), and the Victorian Government's Operational Infrastructure Support Program.

\section{Disclosure Statement}

The authors have no financial relationships relevant to this article and no conflicts of interest to disclose. The study sponsors had no role in (1) the study design; (2) the collection, analysis, and interpretation of data; (3) the writing of the report; and (4) the decision to submit the paper for publication. No honorarium, grant, or other form of payment was given to anyone to produce the paper.

\section{References}

1 Jacobs SE, Berg M, Hunt R, Tarnow-Mordi WO, Inder TE, Davis PG: Cooling for newborns with hypoxic ischaemic encephalopathy. Cochrane Database Syst Rev 2013; 1:CD003311.

2 Edwards AD, Brocklehurst P, Gunn AJ, Halliday $\mathrm{H}$, Juszczak E, Levene M, Strohm B, Thoresen M, Whitelaw A, Azzopardi D: Neurological outcomes at 18 months of age after moderate hypothermia for perinatal hypoxic ischaemic encephalopathy: synthesis and meta-analysis of trial data. BMJ 2010;340: c363.

3 Robertson NJ, Tan S, Groenendaal F, van Bel F, Juul SE, Bennet L, Derrick M, Back SA, Valdez RC, Northington F, Gunn AJ, Mallard C: Which neuroprotective agents are ready for bench to bedside translation in the newborn infant? J Pediatr 2012;160:544.e4-552.e4.

4 Wass CT, Lanier WL, Hofer RE, Scheithauer BW, Andrews AG: Temperature changes of $>$ or $=1^{\circ} \mathrm{C}$ alter functional neurologic outcome and histopathology in a canine model of complete cerebral ischemia. Anesthesiology 1995; 83:325-335.

5 Coimbra C, Boris-Moller F, Drake M, Wieloch T: Diminished neuronal damage in the rat brain by late treatment with the antipyretic drug dipyrone or cooling following cerebral ischemia. Acta Neuropathol 1996;92: 447-453.

6 Coimbra C, Drake M, Boris-Moller F, Wieloch T: Long-lasting neuroprotective effect of postischemic hypothermia and treatment with an anti-inflammatory/antipyretic drug. Evidence for chronic encephalopathic processes following ischemia. Stroke 1996;27: 1578-1585.

7 Campbell K, Meloni BP, Knuckey NW: Combined magnesium and mild hypothermia $\left(35^{\circ} \mathrm{C}\right)$ treatment reduces infarct volumes after permanent middle cerebral artery occlusion in the rat at 2 and 4 , but not $6 \mathrm{~h}$. Brain Res 2008;1230:258-264.
8 Azzimondi G, Bassein L, Nonino F, Fiorani L, Vignatelli L, Re G, D’Alessandro R: Fever in acute stroke worsens prognosis. A prospective study. Stroke 1995;26:2040-2043

9 Terent A, Andersson B: The prognosis for patients with cerebrovascular stroke and transient ischemic attacks. Ups J Med Sci 1981;86: 63-74.

10 Hindfelt B: The prognostic significance of subfebrility and fever in ischaemic cerebral infarction. Acta Neurol Scand 1976;53:7279.

11 DeBow SB, Clark DL, MacLellan CL, Colbourne F: Incomplete assessment of experimental cytoprotectants in rodent ischemia studies. Can J Neurol Sci 2003;30:368-374.

12 Hull GC: Thermoregulation in young mammals; in Whittow GC (ed): Comparative Physiology of Thermoregulation. New York, Academic Press, 1973, pp 167-200.

13 Taylor PM: Oxygen consumption in newborn rats. J Physiol 1960;154:153-168. 
14 Hahn P, Koldovsky O: Prenatal and postnatal development of metabolism and its regulation in mammals (in Czech). Cesk Fysiol 1966;215:437-479.

15 Farrell WJ, Alberts JR: Rat behavioral thermoregulation integrates with nonshivering thermogenesis during postnatal development. Behav Neurosci 2007;121:1333-1341.

16 Fitzgerald LR, Bacon RL: Metabolic rate and thermal regulation in neonatal mice. Anat Rec 1952;113:532.

17 Patel SD, Pierce L, Ciardiello AJ, Vannucci SJ: Neonatal encephalopathy: pre-clinical studies in neuroprotection. Biochem Soc Trans 2014; 42:564-568.

18 Rice JE 3rd, Vannucci RC, Brierley JB: The influence of immaturity on hypoxic-ischemic brain damage in the rat. Ann Neurol 1981;9: 131-141.

19 Romijn HJ, Hofman MA, Gramsbergen A: At what age is the developing cerebral cortex of the rat comparable to that of the full-term newborn human baby? Early Hum Dev 1991; 26:61-67.

20 Wood T, Osredkar D, Puchades M, Maes E, Falck M, Flatebo T, Walloe L, Sabir H, Thoresen M: Treatment temperature and insult severity influence the neuroprotective effects of therapeutic hypothermia. Sci Rep 2016;6: 23430.

21 Galinsky R, Draghi V, Wassink G, Davidson JO, Drury PP, Lear CA, Gunn AJ, Bennet L: Magnesium sulfate reduces EEG activity but is not neuroprotective after asphyxia in preterm fetal sheep. J Cereb Blood Flow Metab 2016, Epub ahead of print.

22 Davidson JO, Rout AL, Wassink G, Yuill CA Zhang FG, Green CR, Bennet L, Gunn AJ: Non-additive effects of delayed connexin hemichannel blockade and hypothermia after cerebral ischemia in near-term fetal sheep. J Cereb Blood Flow Metab 2015;35:2052-2061.

23 Davidson JO, Wassink G, Yuill CA, Zhang FG, Bennet L, Gunn AJ: How long is too long for cerebral cooling after ischemia in fetal sheep? J Cereb Blood Flow Metab 2015;35: 751-758.

24 Laburn HP, Mitchell D, Goelst K: Fetal and maternal body temperatures measured by radiotelemetry in near-term sheep during thermal stress. J Appl Physiol 1992;72:894-900.

25 Galinsky R, Bennet L, Groenendaal F, Lear CA, Tan S, van Bel F, Juul SE, Robertson NJ, Mallard C, Gunn AJ: Magnesium is not consistently neuroprotective for perinatal hypoxia-ischemia in term-equivalent models in preclinical studies: a systematic review. Dev Neurosci 2014;36:73-82.

26 Dobbing J, Sands J: Comparative aspects of the brain growth spurt. Early Hum Dev 1979; 3:79-83.

27 Barlow RM: The foetal sheep: morphogenesis of the nervous system and histochemical aspects of myelination. J Comp Neurol 1969; 135:249-262.
28 Semple BD, Blomgren K, Gimlin K, Ferriero DM, Noble-Haeusslein LJ: Brain development in rodents and humans: identifying benchmarks of maturation and vulnerability to injury across species. Prog Neurobiol 2013; 106-107:1-16.

29 Patel SD, Pierce L, Ciardiello A, Hutton A, Paskewitz S, Aronowitz E, Voss HU, Moore H, Vannucci SJ: Therapeutic hypothermia and hypoxia-ischemia in the term-equivalent neonatal rat: characterization of a translational preclinical model. Pediatr Res 2015;78:264-271.

30 Smith AL, Alexander M, Rosenkrantz TS, Sadek ML, Fitch RH: Sex differences in behavioral outcome following neonatal hypoxia ischemia: insights from a clinical meta-analysis and a rodent model of induced hypoxic ischemic brain injury. Exp Neurol 2014;254: 54-67.

31 Burnsed JC, Chavez-Valdez R, Hossain MS, Kesavan K, Martin LJ, Zhang J, Northington FJ: Hypoxia-ischemia and therapeutic hypothermia in the neonatal mouse brain - a longitudinal study. PLoS One 2015; 10:e0118889.

32 Wyatt JS, Gluckman PD, Liu PY, Azzopardi D, Ballard RA, Edwards AD, Ferriero DM, Polin RA, Robertson CM, Thoresen M, Whitelaw A, Gunn AJ; CoolCap Study Group: Determinants of outcomes after head cooling for neonatal encephalopathy. Pediatrics 2007; 119:912-921.

33 Wright DW, Espinoza TR, Merck LH, Ratcliff JJ, Backster A, Stein DG: Gender differences in neurological emergencies. Part II. A consensus summary and research agenda on traumatic brain injury. Acad Emerg Med 2014;21:1414-1420.

34 Nelson KB, Dambrosia JM, Ting TY, Grether JK: Uncertain value of electronic fetal monitoring in predicting cerebral palsy. $\mathrm{N}$ Engl J Med 1996;334:613-618.

35 Gunn AJ, Bennet L: Timing still key to treating hypoxic ischaemic brain injury. Lancet Neurol 2016;15:126-127.

36 Jacobs SE, Berg M, Hunt R, Tarnow-Mordi WO, Inder TE, Davis PG: Cooling for newborns with hypoxic ischaemic encephalopathy. Cochrane Database Syst Rev 2013;1: CD003311.

37 Geddes R, Vannucci RC, Vannucci SJ: Delayed cerebral atrophy following moderate hypoxia-ischemia in the immature rat. Dev Neurosci 2001;23:180-185.

38 Klahr AC, Nadeau CA, Colbourne F: Temperature control in rodent neuroprotection studies: methods and challenges. Ther Hypothermia Temp Manag 2016, Epub ahead of print.

39 Bai X, Liu S, Yuan L, Xie Y, Li T, Wang L, Wang X, Zhang T, Qin S, Song G, Ge L, Wang $\mathrm{Z}$ : Hydrogen-rich saline mediates neuroprotection through the regulation of endoplasmic reticulum stress and autophagy under hypoxia-ischemia neonatal brain injury in mice. Brain Res 2016;1646:410-417.
40 Broad KD, Fierens I, Fleiss B, Rocha-Ferreira E, Ezzati M, Hassell J, Alonso-Alconada D, Bainbridge A, Kawano G, Ma D, Tachtsidis I, Gressens P, Golay X, Sanders RD, Robertson NJ: Inhaled $45-50 \%$ argon augments hypothermic brain protection in a piglet model of perinatal asphyxia. Neurobiol Dis 2016;87: 29-38.

41 Lafuente H, Pazos MR, Alvarez A, Mohammed N, Santos M, Arizti M, Alvarez FJ, Martinez-Orgado JA: Effects of cannabidiol and hypothermia on short-term brain damage in new-born piglets after acute hypoxia-ischemia. Front Neurosci 2016;10:323.

42 Lai Z, Zhang L, Su J, Cai D, Xu Q: Sevoflurane postconditioning improves long-term learning and memory of neonatal hypoxia-ischemia brain damage rats via the PI3K/AktmPTP pathway. Brain Res 2016;1630:25-37.

43 Li Y, Liu K, Kang ZM, Sun XJ, Liu WW, Mao YF: Helium preconditioning protects against neonatal hypoxia-ischemia via nitric oxide mediated up-regulation of antioxidases in a rat model. Behav Brain Res 2016;300:31-37.

44 Ma Q, Dasgupta C, Li Y, Bajwa NM, Xiong F, Harding B, Hartman R, Zhang L: Inhibition of microRNA-210 provides neuroprotection in hypoxic-ischemic brain injury in neonatal rats. Neurobiol Dis 2016;89:202-212.

45 Mayurasakorn K, Niatsetskaya ZV, Sosunov SA, Williams JJ, Zirpoli H, Vlasakov I, Deckelbaum RJ, Ten VS: DHA but not EPA emulsions preserve neurological and mitochondrial function after brain hypoxia-ischemia in neonatal mice. PLoS One 2016;11: e0160870.

46 Nie X, Lowe DW, Rollins LG, Bentzley J, Fraser JL, Martin R, Singh I, Jenkins D: Sex-specific effects of $\mathrm{N}$-acetylcysteine in neonatal rats treated with hypothermia after severe hypoxia-ischemia. Neurosci Res 2016;108:2433.

47 Reinboth BS, Koster C, Abberger H, Prager S, Bendix I, Felderhoff-Muser U, Herz J: Endogenous hypothermic response to hypoxia reduces brain injury: implications for modeling hypoxic-ischemic encephalopathy and therapeutic hypothermia in neonatal mice. Exp Neurol 2016;283:264-275.

48 Ren X, Ma H, Zuo Z: Dexmedetomidine postconditioning reduces brain injury after brain hypoxia-ischemia in neonatal rats. J Neuroimmune Pharmacol 2016;11:238-247.

49 Xie C, Ginet V, Sun Y, Koike M, Zhou K, Li T, Li H, Li Q, Wang X, Uchiyama Y, Truttmann AC, Kroemer G, Puyal J, Blomgren K, Zhu C: Neuroprotection by selective neuronal deletion of Atg7 in neonatal brain injury. Autophagy 2016;12:410-423.

50 Xu Y, Xue H, Zhao P, Yang Y, Ji G, Yu W, Han $\mathrm{G}$, Ding $\mathrm{M}$, Wang $\mathrm{F}$ : Isoflurane postconditioning induces concentration- and timingdependent neuroprotection partly mediated by the GluR2 AMPA receptor in neonatal rats after brain hypoxia-ischemia. J Anesth 2016; 30:427-436. 
51 Zhao H, Mitchell S, Ciechanowicz S, Savage S, Wang T, Ji X, Ma D: Argon protects against hypoxic-ischemic brain injury in neonatal rats through activation of nuclear factor (erythroid-derived 2)-like 2. Oncotarget 2016;7: 25640-25651.

52 Zhu XY, Ma PS, Wu W, Zhou R, Hao YJ, Niu Y, Sun T, Li YX, Yu JQ: Neuroprotective actions of taurine on hypoxic-ischemic brain damage in neonatal rats. Brain Res Bull 2016; 124:295-305.

53 Allah Yar R, Akbar A, Iqbal F: Creatine monohydrate supplementation for 10 weeks mediates neuroprotection and improves learning/memory following neonatal hypoxia ischemia encephalopathy in female albino mice. Brain Res 2015;1595:92-100.

54 Alonso-Alconada D, Broad KD, Bainbridge A, Chandrasekaran M, Faulkner SD, Kerenyi A, Hassell J, Rocha-Ferreira E, Hristova M, Fleiss B, Bennett K, Kelen D, Cady E, Gressens $\mathrm{P}$, Golay X, Robertson NJ: Brain cell death is reduced with cooling by $3.5^{\circ} \mathrm{C}$ to $5^{\circ} \mathrm{C}$ but increased with cooling by $8.5^{\circ} \mathrm{C}$ in a piglet asphyxia model. Stroke 2015;46:275-278.

55 Arteaga O, Revuelta M, Uriguen L, Alvarez A, Montalvo $\mathrm{H}$, Hilario E: Pretreatment with resveratrol prevents neuronal injury and cognitive deficits induced by perinatal hypoxiaischemia in rats. PLoS One 2015;10:e0142424.

56 Charles MS, Drunalini Perera PN, Doycheva DM, Tang J: Granulocyte-colony stimulating factor activates JAK2/PI3K/PDE3B pathway to inhibit corticosterone synthesis in a neonatal hypoxic-ischemic brain injury rat model. Exp Neurol 2015;272:152-159.

57 Chen W, Xu B, Xiao A, Liu L, Fang X, Liu R, Turlova E, Barszczyk A, Zhong X, Sun CL, Britto LR, Feng ZP, Sun HS: TRPM7 inhibitor carvacrol protects brain from neonatal hypoxic-ischemic injury. Mol Brain 2015;8:11.

58 Iqbal S, Ali M, Iqbal F: Long term creatine monohydrate supplementation, following neonatal hypoxic ischemic insult, improves neuromuscular coordination and spatial learning in male albino mouse. Brain Res 2015;1603:76-83.

59 Koyuncuoglu T, Turkyilmaz M, Goren B, Cetinkaya M, Cansev M, Alkan T: Uridine protects against hypoxic-ischemic brain injury by reducing histone deacetylase activity in neonatal rats. Restor Neurol Neurosci 2015;33: 777-784.

60 Kwak M, Lim S, Kang E, Furmanski O, Song $\mathrm{H}$, Ryu YK, Mintz CD: Effects of neonatal hypoxic-ischemic injury and hypothermic neuroprotection on neural progenitor cells in the mouse hippocampus. Dev Neurosci 2015;37: 428-439.

61 Li X, Zhao H, Tan X, Kostrzewa RM, Du G, Chen Y, Zhu J, Miao Z, Yu H, Kong J, Xu X: Inhibition of connexin 43 improves functional recovery after ischemic brain injury in neonatal rats. Glia 2015;63:1553-1567.

62 Liu X, Dingley J, Scull-Brown E, Thoresen M: Adding $5 \mathrm{~h}$ delayed xenon to delayed hypothermia treatment improves long-term func- tion in neonatal rats surviving to adulthood. Pediatr Res 2015;77:779-783.

63 Min JW, Hu JJ, He M, Sanchez RM, Huang WX, Liu YQ, Bsoul NB, Han S, Yin J, Liu WH, $\mathrm{He} \mathrm{XH}$, Peng BW: Vitexin reduces hypoxiaischemia neonatal brain injury by the inhibition of HIF-1alpha in a rat pup model. Neuropharmacology 2015;99:38-50.

64 Park D, Shin K, Choi EK, Choi Y, Jang JY, Kim J, Jeong HS, Lee W, Lee YB, Kim SU, Joo SS, Kim YB: Protective effects of N-acetyl-Lcysteine in human oligodendrocyte progenitor cells and restoration of motor function in neonatal rats with hypoxic-ischemic encephalopathy. Evidence-based complementary and alternative medicine: eCAM 2015;2015: 764251.

65 Peterson BL, Won S, Geddes RI, Sayeed I, Stein DG: Sex-related differences in effects of progesterone following neonatal hypoxic brain injury. Behav Brain Res 2015;286:152165.

66 Smith AL, Garbus H, Rosenkrantz TS, Fitch RH: Sex differences in behavioral outcomes following temperature modulation during induced neonatal hypoxic ischemic injury in rats. Brain Sci 2015;5:220-240.

67 Sosunov SA, Ameer X, Niatsetskaya ZV, Utkina-Sosunova I, Ratner VI, Ten VS: Isoflurane anesthesia initiated at the onset of reperfusion attenuates oxidative and hypoxicischemic brain injury. PLoS One 2015; 10:e0120456.

68 Sun HS, Xu B, Chen W, Xiao A, Turlova E, Alibraham A, Barszczyk A, Bae CY, Quan Y, Liu B, Pei L, Sun CL, Deurloo M, Feng ZP: Neuronal K(ATP) channels mediate hypoxic preconditioning and reduce subsequent neonatal hypoxic-ischemic brain injury. Exp Neurol 2015;263:161-171.

69 Takenouchi T, Sugiura Y, Morikawa T, Nakanishi T, Nagahata Y, Sugioka T, Honda K, Kubo A, Hishiki T, Matsuura T, Hoshino T, Takahashi T, Suematsu M, Kajimura M: Therapeutic hypothermia achieves neuroprotection via a decrease in acetylcholine with a concurrent increase in carnitine in the neonatal hypoxia-ischemia. J Cereb Blood Flow Metab 2015;35:794-805.

70 Tan X, Chen Y, Li J, Li X, Miao Z, Xin N, Zhu J, Ge W, Feng Y, Xu X: The inhibition of Cdk5 activity after hypoxia/ischemia injury reduces infarct size and promotes functional recovery in neonatal rats. Neuroscience 2015;290:552560.

71 Thatipamula S, Al Rahim M, Zhang J, Hossain MA: Genetic deletion of neuronal pentraxin 1 expression prevents brain injury in a neonatal mouse model of cerebral hypoxiaischemia. Neurobiol Dis 2015;75:15-30.

72 Wang Z, Feng C, Zhao H, Ren X, Peng S, Zuo $\mathrm{Z}$ : Autoregulation of inducible nitric oxide synthase expression by RNA interference provides neuroprotection in neonatal rats. Theranostics 2015;5:504-514.

73 Zhao P, Zhou R, Li HN, Yao WX, Qiao HQ, Wang SJ, Niu Y, Sun T, Li YX, Yu JQ: Oxy- matrine attenuated hypoxic-ischemic brain damage in neonatal rats via improving antioxidant enzyme activities and inhibiting cell death. Neurochem Int 2015;89:17-27.

74 Bolouri H, Savman K, Wang W, Thomas A, Maurer N, Dullaghan E, Fjell CD, Ek CJ, Hagberg H, Hancock RE, Brown KL, Mallard C: Innate defense regulator peptide 1018 protects against perinatal brain injury. Ann Neurol 2014;75:395-410.

75 Charriaut-Marlangue C, Nguyen T, Bonnin P, Duy AP, Leger PL, Csaba Z, Pansiot J, Bourgeois T, Renolleau S, Baud O: Sildenafil mediates blood-flow redistribution and neuroprotection after neonatal hypoxia-ischemia. Stroke 2014;45:850-856.

76 Gonzalez-Rodriguez PJ, Li Y, Martinez F, Zhang L: Dexamethasone protects neonatal hypoxic-ischemic brain injury via L-PGDSdependent PGD2-DP1-pERK signaling pathway. PLoS One 2014;9:e114470.

77 Han W, Sun Y, Wang X, Zhu C, Blomgren K: Delayed, long-term administration of the caspase inhibitor Q-VD-OPh reduced brain injury induced by neonatal hypoxia-ischemia. Dev Neurosci 2014;36:64-72.

78 Hsu YC, Chang YC, Lin YC, Sze CI, Huang CC, Ho CJ: Cerebral microvascular damage occurs early after hypoxia-ischemia via nNOS activation in the neonatal brain. J Cereb Blood Flow Metab 2014;34:668-676.

79 Kaandorp JJ, Derks JB, Oudijk MA, Torrance HL, Harmsen MG, Nikkels PG, van Bel F, Visser GH, Giussani DA: Antenatal allopurinol reduces hippocampal brain damage after acute birth asphyxia in late gestation fetal sheep. Reprod Sci 2014;21:251-259.

80 Kilicdag H, Daglioglu YK, Erdogan S, Zorludemir S: Effects of caffeine on neuronal apoptosis in neonatal hypoxic-ischemic brain injury. J Matern Fetal Neonatal Med 2014;27: 1470-1475.

81 Kim GS, Cho S, Nelson JW, Zipfel GJ, Han $\mathrm{BH}$ : TrkB agonist antibody pretreatment enhances neuronal survival and long-term sensory motor function following hypoxic ischemic injury in neonatal rats. PLoS One 2014; 9:e88962.

82 Lange S, Rocha-Ferreira E, Thei L, Mawjee P, Bennett K, Thompson PR, Subramanian V, Nicholas AP, Peebles D, Hristova M, Raivich G: Peptidylarginine deiminases: novel drug targets for prevention of neuronal damage following hypoxic ischemic insult (HI) in neonates. J Neurochem 2014; 130:555-562.

83 Lin EP, Miles L, Hughes EA, McCann JC, Vorhees CV, McAuliffe JJ, Loepke AW: A combination of mild hypothermia and sevoflurane affords long-term protection in a modified neonatal mouse model of cerebral hypoxia-ischemia. Anesth Analg 2014;119: 1158-1173.

84 Sabir H, Walloe L, Dingley J, Smit E, Liu X, Thoresen M: Combined treatment of xenon and hypothermia in newborn rats - additive or synergistic effect? PLoS One 2014;9:e109845.
Temperature Control and

Neuroprotection
Dev Neurosci 2017;39:7-22 DOI: $10.1159 / 000452859$ 
85 Threlkeld SW, Gaudet CM, La Rue ME, Dugas E, Hill CA, Lim YP, Stonestreet BS: Effects of inter-alpha inhibitor proteins on neonatal brain injury: age, task and treatment dependent neurobehavioral outcomes. Exp Neurol 2014;261:424-433.

86 Tskitishvili E, Nisolle M, Munaut C, Pequeux C, Gerard C, Noel A, Foidart JM: Estetrol attenuates neonatal hypoxic-ischemic brain injury. Exp Neurol 2014;261:298-307.

87 Xie C, Zhou K, Wang X, Blomgren K, Zhu C: Therapeutic benefits of delayed lithium administration in the neonatal rat after cerebral hypoxia-ischemia. PLoS One 2014;9:e107192.

88 Zhao P, Ji G, Xue H, Yu W, Zhao X, Ding M, Yang Y, Zuo Z: Isoflurane postconditioning improved long-term neurological outcome possibly via inhibiting the mitochondrial permeability transition pore in neonatal rats after brain hypoxia-ischemia. Neuroscience 2014; 280:193-203.
89 Zhu J, Wang B, Lee JH, Armstrong JS, Kulikowicz E, Bhalala US, Martin LJ, Koehler RC, Yang ZJ: Additive neuroprotection of a 20-HETE inhibitor with delayed therapeutic hypothermia after hypoxia-ischemia in neonatal piglets. Dev Neurosci 2015;37:376389.

90 Sabir H, Osredkar D, Maes E, Wood T, Thoresen $\mathrm{M}$ : Xenon combined with therapeutic hypothermia is not neuroprotective after severe hypoxia-ischemia in neonatal rats. PLoS One 2016;11:e0156759.

91 Bonestroo HJ, Nijboer $\mathrm{CH}$, van Velthoven CT, van Bel F, Heijnen CJ: The neonatal brain is not protected by osteopontin peptide treatment after hypoxia-ischemia. Dev Neurosci 2015;37:142-152.

92 Hoque N, Liu X, Chakkarapani E, Thoresen M: Minimal systemic hypothermia combined with selective head cooling evaluated in a pig model of hypoxia-ischemia. Pediatr Res 2015; 77:674-680.
93 Osredkar D, Sabir H, Falck M, Wood T, Maes E, Flatebo T, Puchades M, Thoresen M: Hypothermia does not reverse cellular responses caused by lipopolysaccharide in neonatal hypoxic-ischaemic brain injury. Dev Neurosci 2015;37:390-397.

94 Wang B, Armstrong JS, Lee JH, Bhalala U, Kulikowicz E, Zhang H, Reyes M, Moy N, Spicer D, Zhu J, Yang ZJ, Koehler RC, Martin LJ, Lee JK: Rewarming from therapeutic hypothermia induces cortical neuron apoptosis in a swine model of neonatal hypoxic-ischemic encephalopathy. J Cereb Blood Flow Metab 2015;35:781-793.

95 Osredkar D, Thoresen M, Maes E, Flatebo T, Elstad M, Sabir H: Hypothermia is not neuroprotective after infection-sensitized neonatal hypoxic-ischemic brain injury. Resuscitation 2014;85:567-572. 